\title{
Scalable Keyword Search on Large RDF Data
}

\author{
Wangchao Le, Feifei Li, Anastasios Kementsietsidis, Songyun Duan
}

\begin{abstract}
Keyword search is a useful tool for exploring large RDF datasets. Existing techniques either rely on constructing a distance matrix for pruning the search space or building summaries from the RDF graphs for query processing. In this work, we show that existing techniques have serious limitations in dealing with realistic, large RDF data with tens of millions of triples. Furthermore, the existing summarization techniques may lead to incorrect/incomplete results. To address these issues, we propose an effective summarization algorithm to summarize the RDF data. Given a keyword query, the summaries lend significant pruning powers to exploratory keyword search and result in much better efficiency compared to previous works. Unlike existing techniques, our search algorithms always return correct results. Besides, the summaries we built can be updated incrementally and efficiently. Experiments on both benchmark and large real RDF data sets show that our techniques are scalable and efficient.
\end{abstract}

\section{INTRODUCTION}

The RDF (Resource Description Framework) is the de-facto standard for data representation on the Web. So, it is no surprise that we are inundated with large amounts of rapidly growing RDF data from disparate domains. For instance, the Linked Ope n Data (LOD) initiative integrates billions of entities from hundreds of sources. Just one of these sources, the DBpedia dataset, describes more than 3.64 million things using more than 1 billion RDF triples; and it contains numerous keywords, as shown in Figure 1 .

Keyword search is an important tool for exploring and searching large data corpuses whose structure is either unknown, or constantly changing. So, keyword search has already been studied in the context of relational databases [3], [7], [15], [19], XML documents [8], [22], and more recently over graphs [14], [17] and RDF data [11], [23]. However, existing solutions for RDF data have limitations. Most notably, these solutions suffer from: (i) returning incorrect answers, i.e., the keyword search returns answers that do not correspond to real subgraphs or misses valid matches from the underlying RDF data; (ii) inability to scale to handle typical RDF datasets with tens of millions of triples. Consider the results from two representative solutions [14], [23], as shown in Figures 2 and 3 . Figure 2 shows the query results on three different datasets using the solution specifically designed for RDF in [23], the Schema method. While this solution may perform well on datasets that have regular topological structure (e.g., DBLP), it returns incorrect answers for others (e.g., LUBM [13] etc.) when compared to a naive, but Exact method. On the other hand, classical techniques [14] proposed for general graphs can be used for RDF data, but they assume a distance matrix built on the data, which makes it prohibitively expensive to apply to large RDF dataset as shown in Figure 3

Motivated by these observations, we present a comprehensive study to address the keyword search problem over big

Wangchao Le and Feifei Li are with the School of Computing, University of Utah. E-mail: $\{$ lew, lifeifei\}@cs.utah.edu .

Anastasios Kementsietsidis and Songyun Duan are with IBM Thomas J. Watson Research Center. E-mail: tasosk@ca.ibm.com, sduan@us.ibm.com.

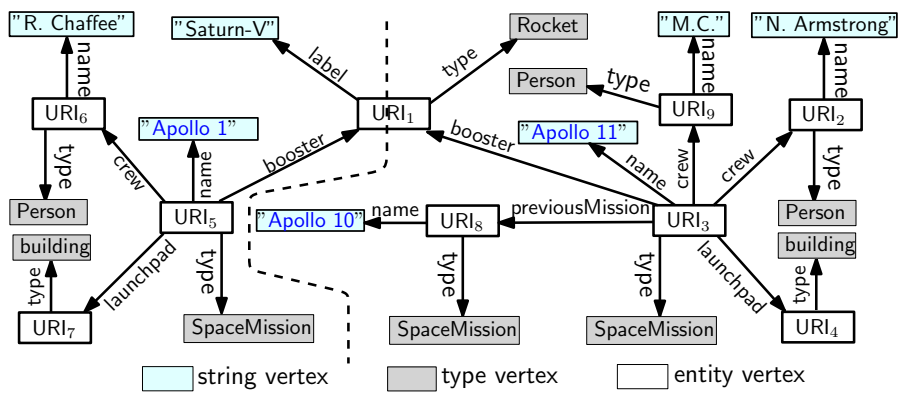

Fig. 1. Keywords in a sample from DBpedia.
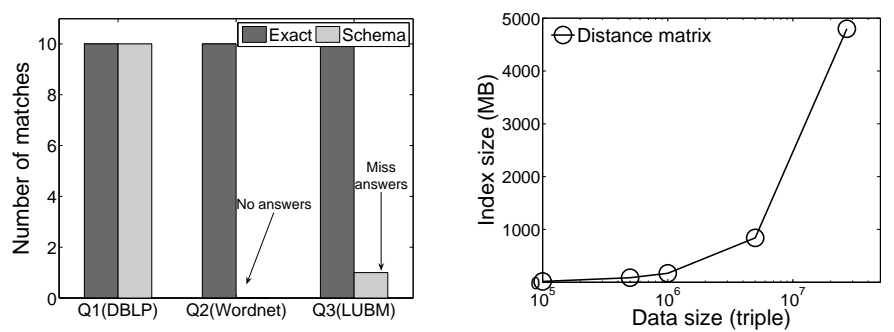

Fig. 2. Schema method [23]. Fig. 3. Distance matrix [14].

RDF data. Our goal is to design a scalable and exact solution that handles realistic RDF datasets with tens of millions of triples. To address the scalability issues, our solution builds a new, succinct and effective summary from the underlying RDF graph based on its types. Given a keyword search query, we use the summary to prune the search space, leading to much better efficiency compared to a baseline solution. To summarize, our contributions are:

- We identify and address limitations in the existing, stateof-the-art methods for keyword search in RDF data [23]. We show that these limitations could lead to incomplete and incorrect answers in real RDF datasets. We propose a new, correct baseline solution based on the backward search idea.

- We develop efficient algorithms to summarize the structure of RDF data, based on the types in RDF graphs, and use it to speed up the search. Compared to previous works that also build summary [11], [23], our technique uses different intuitions, which is more scalable and lends significant pruning power without sacrificing the soundness of the result. Further, 
our summary is light-weight and updatable.

- Our experiments on both benchmark and large real RDF datasets show that our techniques are much more scalable and efficient in correctly answering keyword search queries for realistic RDF workloads than the existing methods.

In what follows, we formulate the keyword search problem on RDF data in Section 2, survey related work in Section 3 . present our solutions in Sections 4 to 7 , show experimental results in Section 8, and conclude in Section 9 Table 1 list the frequently used symbols.

\section{Preliminaries}

An RDF dataset is a graph (RDF graph) composed by triples, where a triple is formed by subject, predicate and object in that order. When such ordering is important semantically, a triple is regarded as a directed edge (the predicate) connecting two vertices (from subject to object). Thus, an RDF dataset can be alternatively viewed as a directed graph, as shown by the arrows in Figure 11. W3C has provided a set of unified vocabularies (as part of the RDF standard) to encode the rich semantics. From these, the rdfs:type predicate (or type for short) is particularly useful to our problem (see Section 5, since it provides a classification of vertices of an RDF graph into different groups. For instance in Figure 1, the entity $\mathrm{URI}_{3}$ has type SpaceMission. Formally, we view an RDF dataset as an RDF graph $G=(V, E)$ where

- $V$ is the union of disjoint sets, $V_{E}, V_{T}$ and $V_{W}$, where $V_{E}$ is the set of entity vertices (i.e.,URIs), $V_{T}$ is the set of type vertices, and $V_{W}$ is a set of keyword vertices.

- $E$ is the union of disjoint sets, $E_{R}, E_{A}$, and $E_{T}$ where $E_{R}$ is the set of entity-entity edges (i.e., connecting two vertices in $\left.V_{E}\right), E_{A}$ is the set of entity-keyword edges (i.e., connecting an entity to a keyword), and $E_{T}$ is the set entity-type edges (i.e., connecting an entity to a type).

\begin{tabular}{|c|c|c|c|c|}
\hline URI $_{5}$ SpaceMission & booster & URI $_{1} \quad$ Rocket & booster & URI $_{3}$ SpaceMission \\
\hline "Apollo 1" & & "Saturn-V" & & "Apollo 11" \\
\hline
\end{tabular}

\section{Fig. 4. Condensed view: combining vertices.}

For example in Figure 1, all gray vertices are type vertices while entity vertices are in white. Each entity vertex also has associated keyword vertices (in cyan). The division on vertices results in a corresponding division on the RDF predicates, which leads to the classification of the edge set $E$ discussed earlier. Clearly, the main structure of an RDF graph is captured by the entity-entity edges represented by the set $E_{R}$. As such, an alternative view is to treat an entity vertex and its associated type and keyword vertices as one vertex. For example, the entity vertices $U_{R} I_{5}, U R I_{1}$ and $U^{2} I_{3}$ from Figure 1 , with their types and keywords, can be viewed as the structure in Figure 4.

In general, for an RDF graph $G=\{V, E\}$, we will refer this as the condensed view of $G$, denoted as $G_{c}=\left\{V_{E}^{\prime}, E_{R}\right\}$. While $\left|V_{E}^{\prime}\right| \equiv\left|V_{E}\right|$, every vertex $v^{\prime} \in V_{E}^{\prime}$ contains not only the entity value of a corresponding vertex $v \in V_{E}$, but also the associated keyword(s) and type(s) of $v$. For the ease of presentation, hereafter we associate a single keyword and a single type to each entity. Our techniques can be efficiently

\begin{tabular}{|l|l|}
\hline Symbol & Description \\
\hline$G\{V, E\}$ & the condensed view of an RDF graph. \\
\hline $\mathcal{A}(q)$ & top- $k$ answers for a query. \\
\hline$r$ & answer root. \\
\hline$w_{i}$ & the $i$-th keyword. \\
\hline$d(x, y)$ & graph distance between node $x$ and node $y$. \\
\hline$C(q)$ & a set of candidate answers. \\
\hline$\alpha$ & used to denote the $\alpha$-hop neighborhoods. \\
\hline$g$ & an answer subgraph of $G$. \\
\hline$S$ & the summaries of $\mathcal{P}$. \\
\hline$W i_{i}$ & the set of nodes containing keyword $w_{i}$. \\
\hline $\mathcal{P}$ & partitions. \\
\hline$M$ & for bookkeeping the candidate answers. \\
\hline$h(v, \alpha), h$ & the $\alpha$-hop neighborhoods of $v$, a partition. \\
\hline$h_{t}(v, \alpha), h_{t}$ & the covering tree of $h(v, \alpha)$, a covering tree. \\
\hline $\mathcal{S}$ & a path represented by a sequence of partitions. \\
\hline$d_{l}, d_{u}$ & the lower and upper bounds (for a path). \\
\hline$\sigma$ & a one-to-many mapping in converting $h$ to $h_{t}$. \\
\hline$\Sigma$ & a set of $\sigma$ 's from a partition $h$. \\
\hline
\end{tabular}

TABLE 1: Frequently used notations.

extended to handle the general cases. Also for simplicity, hereafter, we use $G=\{V, E\}$ to represent the condensed view of an RDF graph.

We assume that readers are familiar with the SPARQL query lanaguge; a brief review of SPARQL is also provided in the online appendix, in Section 11 .

\subsection{Problem statement}

Intuitively, a keyword query against an RDF graph looks for (smallest) subgraphs that contain all the keywords. Given an RDF graph $G=\{V, E\}$, for any vertex $v \in V$, denote the keyword stored in $v$ as $w(v)$. For the ease of presentation, we assume each vertex contains a single keyword. However the solutions we have developed can be seamlessly applied to general cases where a vertex has multiple keywords or no keywords. Formally, a keyword search query $q$ against an RDF data set $G=\{V, E\}$ is defined by $m$ unique keywords $\left\{w_{1}, w_{2}, \ldots, w_{m}\right\}$. A set of vertices $\left\{r, v_{1}, \ldots, v_{m}\right\}$ from $V$ is a qualified candidate when:

- $r \in V$ is called a root answer node which is reachable by $v_{i} \in V$ for $i \in[1, m]$

- $w\left(v_{i}\right)=w_{i}$.

If we define the answer for $q$ as $\mathcal{A}(q)$ and the set of all qualified candidates in $G$ with respect to $q$ as $C(q)$, then

$$
\mathcal{A}(q)=\underset{g \in C(q)}{\arg \min } s(g), \text { and } s(g)=\sum_{r, v_{i} \in g, i=1 . . m} d\left(r, v_{i}\right)
$$

where $d\left(r, v_{i}\right)$ is the graph distance between vertices $r$ and $v_{i}$ (when treating $G$ as an undirected graph). Intuitively, this definition looks for a subgraph in an RDF graph that has minimum length to connect all query keywords from a root node $r$. In prior works concerning keyword search in RDF data, the graph distance of $d\left(v_{1}, v_{2}\right)$ is simply the shortest path between $v_{1}$ and $v_{2}$ in $G$, where each edge is assigned a weight of 1 (in the case of general graph [14], the weight of each edge could be different). Note that if $v_{1}$ and $v_{2}$ belong to disconnected parts of $G$, then $d\left(v_{1}, v_{2}\right)=+\infty$. Also note that this metric (i.e., eq. 11] is proposed by [14] and has been used by prior work on keyword search in RDF data [11], [23]. 
This definition has a top- $k$ version, where the query asks for the top $k$ qualified candidates from $C(q)$. Let the score of a qualified candidate $g \in C(q)$ defined as $s(g)$ in (1), then we can rank all qualified candidates in $C(q)$ in an ascending order of their scores, and refer to the $i$ th ranked qualified candidate as $A(q, i)$. The answer to a top- $k$ keyword search query $q$ is an ordered set $\mathcal{A}(q, k)=\{A(q, 1), \ldots, A(q, k)\} . \mathcal{A}(q)$ is a special case when $k=1$, and $\mathcal{A}(q)=\mathcal{A}(q, 1)$. Lastly, we adopt the same assumption as in the prior works [14], [23] that the answer roots in $\mathcal{A}$ are distinct.

Unless otherwise specified, all proofs in this paper appear in the online appendix, in Section 12.

\section{Related Work}

For keyword search on generic graphs, many techniques [14], [17] assume that graphs fit in memory, an assumption that breaks for big RDF graphs. For instance, the approaches in [14], [17] maintain a distance matrix for all vertex pairs, and clearly do not scale for graphs with millions of vertices. Furthermore, these works do not consider how to handle updates. A typical approach used here for keyword-search is backward search. Backward search when used to find a Steiner tree in the data graph is NP-hard. He et al [14] proposed a tractable problem that does not aim to find a Steiner tree and can be answered by using backward search. In this work we extend this problem to large RDF graphs with rigorous analysis, and without depending on the distance matrix.

Techniques for summarizing large graph data to support keyword search were also studied [9]. The graph data are first partitioned into small subgraphs by heuristics. In this version of the problem, the authors assumed edges across the boundaries of the partitions are weighted. A partition is treated as a supernode and edges crossing partitions are superedges. The supernodes and superedges form a new graph, which is considered as a summary the underlying graph data. By recursively performing partitioning and building summaries, a large graph can be eventually summarized with a small summary and fit into memory for query processing. During query evaluation, the correspondent supernodes containing the keywords being queried are unfolded and the respective portion of graph are fetched from external memory for query processing. This approach is proposed for generic graphs, and cannot be extended for RDF data, as edges in RDF data are predicates and are not weighed. Furthermore, the portion of the graph that does not contain any keyword being queried is still useful in query evaluation, therefore, this approach cannot be applied to address our problem. A summary built in this manner is not updatable.

Keyword search for RDF data has been recently studied in [23], which adopted the popular problem defintion from [14] as we do in this paper. In this approach, a schema to represent the relations among entities of distinct types is summarized from the RDF data set. Backward search is first applied on the schema/summary of the data to identify promising relations which could have all the keywords being queried. Then, by translating these relations into search patterns in SPARQL queries and executing them against the RDF data, the actual subgraphs are retrived.

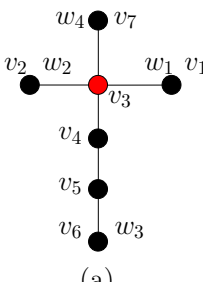

(a) (b)

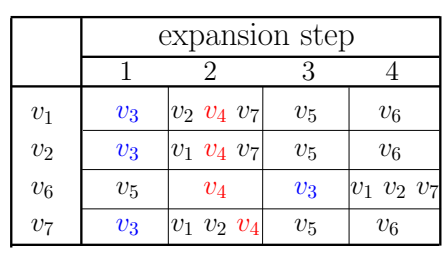

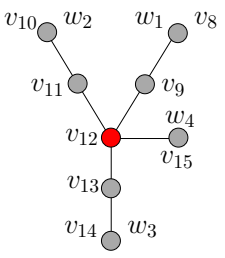

(c)

\section{Fig. 5. Backward search.}

The proposed summarization process in [23] has a limitation: it bundles all the entities of the same type into one node in its summary, which loses too much information in data as to how one type of entities are connected to other types of entities. As a result, this approach could generates erroneous results (both false positives and false negatives). We have given one example in Figure 2 As another example, consider Figure 1. In this approach, all vertices of the type SpaceMission are represented by one node named SpaceMission in the summary. Then, summarizing the predicate previousMission connecting $U R I_{3}$ and $U R I_{8}$ results in a self-loop over the node SpaceMission in the summary, which is incorrect as such a loop does not exist in the data. To be more concrete, when a user asks for all the space missions together with their previous missions, the search pattern in SPARQL would be \{?x Previousmission ?x. ?x type spacemission.\}, which is resultless in DBpedia. Furthermore, such a summary does not support updates. While we also built our summarization using type information, our summarization process uses different intuitions, which guarantees (a) the soundness of the results; and (b) the support of efficient updates.

There are other works related to keyword search on graphs. In [18], a 3-in-1 method is proposed to answer keyword search on structured, semi-structured and unstructured data. The idea is to encode the heterogeneous relations as a graph. Similar to [14], [17], it also needs to maintain a distance matrix. An orthogonal problem to keyword search on graph is the study of different ranking functions. This problem is studied in [11], [12]. In this work, we adopt the standard scoring function in previous work in RDF [23] and generic graphs [14].

\section{The Baseline Method}

A baseline solution is based on the "backward search" heuristic. Intuitively, the "backward search" starts simultaneously from each vertex in the graph $G$ that corresponds to a query keyword, and expands to its neighboring nodes recursively until a candidate answer is generated. A termination condition is used to determine whether the search is complete.

The state-of-the-art keyword search method on RDF graphs [23] has applied the backward search idea. Their termination condition is to stop the search whenever the expansions originating from $m$ vertices $\left\{v_{1}, \ldots, v_{m}\right\}$ (each corresponding to a distinct query keyword) meet at a node $r$ for the first time, where $\left\{r, v_{1}, \ldots, v_{m}\right\}$ is returned as the answer. Unfortunately, this termination condition is incorrect.

Counter example. Consider the graph in Figure 5 (a) and a top-1 query $q=\left\{w_{1}, w_{2}, w_{3}, w_{4}\right\}$. The steps for the four backward expansions performed on Figure 5(a) are shown 
in Figure $5 \mathrm{~b}$ ). Using the above termination condition, the backward expansions from the four vertices $\left\{v_{1}, v_{2}, v_{6}, v_{7}\right\}$ covering the query keywords $\left\{w_{1}, w_{2}, w_{3}, w_{4}\right\}$ meet for the first time in the second iteration, so the candidate answer $g=\left\{r=v_{4}, v_{1}, v_{2}, v_{6}, v_{7}\right\}$ is returned and $s(g)=8$. However, if we continue to the next iteration, the four expansions will meet again at $v_{3}$, with $g^{\prime}=\left\{r=v_{3}, v_{1}, v_{2}, v_{6}, v_{7}\right\}$ and $s\left(g^{\prime}\right)=6$, which is the best answer. One may argue that the graph covering the query keywords is still correctly identified. However, it will be problematic if we also consider the graph in Figure 5(c) as input for the search. There, the best possible answer would be $g^{\prime \prime}=\left\{r=v_{12}, v_{8}, v_{10}, v_{14}, v_{15}\right\}$ and $s\left(g^{\prime \prime}\right)=7<s(g)$. Hence, $g^{\prime \prime}$ will be declared as the top1 answer for $q$ instead of $g^{\prime}$, which is clearly incorrect. Furthermore, later we will explain that even if we fix this error in the terminating condition, their method [23] may still return incorrect results due to the limitations in the summary it builds, as shown by the results in Figure 2

The correct termination. Next, we show the correct termination condition for the backward search on RDF data. The complete algorithm appears in Algorithm 1 .

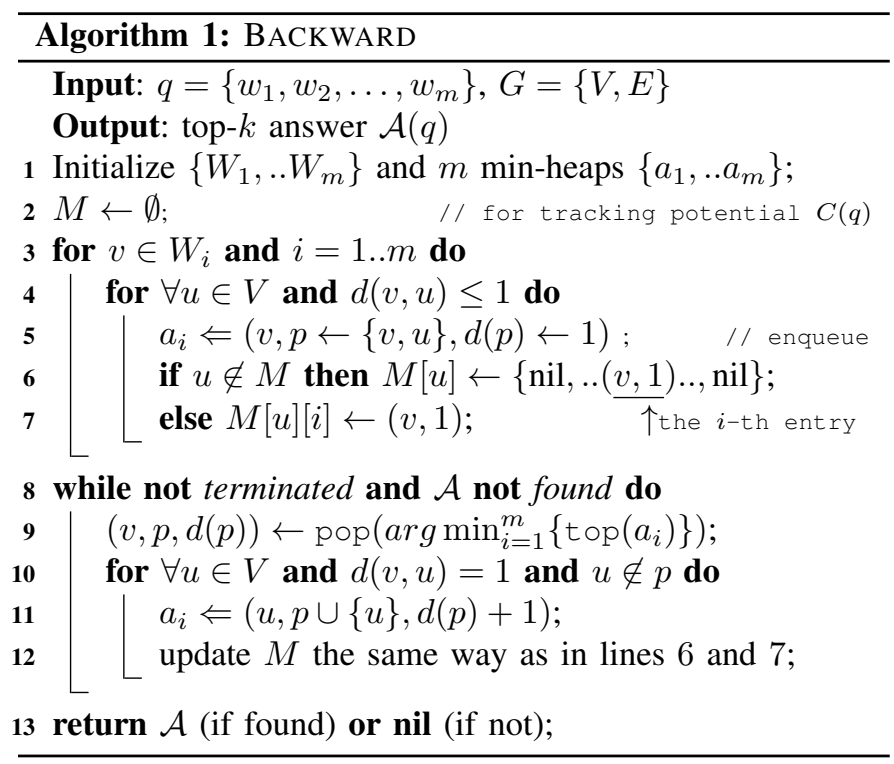

Data structures. Given $q=\left\{w_{1}, \ldots, w_{m}\right\}$ and a (condensed) RDF graph $G=\{V, E\}$, we use $W_{i}$ to denote the set of vertices in $V$ containing the keyword $w_{i}$ (line 1 ). We initialize $m$ empty priority queues (e.g., min-heaps) $\left\{a_{1}, . . a_{m}\right\}$, one for each query keyword (line 1 ). We also maintain a set $M$ of elements (line 2), one for each distinct node we have explored so far in the backward expansion to track the state of the node, i.e., what keywords are reachable to the node and their best known distances. In what follows, we use $M[v]$ to indicate the bookkeeping for the node $v$. Specifically, in each element of $M$, we store a list of $m$ (vertex, distance) pairs. A (vertex, distance) pair in the $j$-th entry of $M[v]$ indicates a (shortest) path from vertex that reaches $v$ in distance hops and it is the shortest possible path starting from any instance of $w_{j}$ (recall that there could have multiple copies of $w_{j}$ in $G$ ). Next, we also use $M[v][j]$ to indicate the $j$-th pair in $M[v]$. For instance in Figure 5 a), consider an element $M\left[v_{3}\right]=\left\{\left(v_{1}, 1\right),\left(v_{2}, 1\right)\right.$, nil, $\left.\left(v_{7}, 1\right)\right\}$ in $M$. The entry indicates that $v_{3}$ has been reached by three expansions from vertices $v_{1}, v_{2}$ and $v_{7}$, containing keywords $w_{1}, w_{2}$ and $w_{4}$ respectively - each can reach $v_{3}$ in one hop. However, $v_{3}$ has not been reached by any expansion from any vertex containing $w_{3}$ yet.

The algorithm. With the structures in place, the algorithm proceeds in iterations. In the first iteration (lines 3-7), for each vertex $v$ from $W_{i}$ and every neighbor $u$ of $v$ (including $v$ itself), we add an entry $(v, p \leftarrow\{v, u\}, d(p))$ to the priority queue $a_{i}$ (entries are sorted in the ascending order of $d(p)$ where $p$ stands for a path and $d(p)$ represents its length). Next, we look for the newly expanded node $u$ in $M$. If $u \in M$, we simply replace $M[u][i]$ with $(v, d(\mathrm{p}))$ (line 7 ). Otherwise, we initialize an empty element for $M[u]$ and set $M[u][i]=(v, d(\mathrm{p}))$ (line 6$)$. We repeat this process for all $W_{i}$ 's for $i=1 . . m$.

In the $j$-th $(j>1)$ iteration of our algorithm (lines 8-12), we pop the smallest top entry of $\left\{a_{1} . . a_{m}\right\}$ (line 9 ), say an entry $(v, p=\{v, \ldots, u\}, d(p))$ from the queue $a_{i}$. For each neighboring node $u^{\prime}$ of $u$ in $G$ such that $u^{\prime}$ is not in $p$ yet (i.e., not generating a cycle), we push an entry $\left(v, p \cup\left\{u^{\prime}\right\}, d(p)+1\right)$ back to the queue $a_{i}$ (line 11). We also update $M$ with $u^{\prime}$ similarly as above (line 12 ). This concludes the $j$-th iteration.

In any step, if an entry $M[u]$ for a node $u$ has no nil pairs in its list of $m$ (vertex, distance) pairs, this entry identifies a candidate answer and $u$ is a candidate root. Due to the property of the priority queue and the the fact that all edges have a unit weight, the paths in $M[u]$ are the shortest paths to $u$ from $m$ distinct query keywords. Denote the graph concatenated by the list of shortest paths in $M[u]$ as $g$. We have:

Lemma $1 g=\left\{r=u, v_{\ell_{1}}, \ldots, v_{\ell_{m}}\right\}$ is a candidate answer with $s(g)=\sum_{i=1}^{m} d\left(u, v_{\ell_{i}}\right)$.

A node $v$ is not fully explored if it has not been reached by at least one of the query keywords. Denote the set of vertices that are not fully explored as $V_{t}$, and the top entries from the $m$ expansion queues (i.e., min-heaps) $a_{1} . . a_{m}$ as $\left(v_{1}, p_{1}, d\left(p_{1}\right)\right), \ldots,\left(v_{m}, p_{m}, d\left(p_{m}\right)\right)$. Consider two cases: (i) an unseen vertex, i.e., $v \notin M$, will become the answer root; (ii) a seen but not fully expanded vertex $v \in M$ will become the answer root. The next two lemmas bound the optimal costs for these two cases respectively. For the first case, Lemma 2 provides a lower bound for the best potential cost.

Lemma 2 Denote the best possible candidate answer as $g_{1}$, and a vertex $v \notin M$ as the answer root of $g_{1}$. Then it must have $s\left(g_{1}\right)>\sum_{i=1}^{m} d\left(p_{i}\right)$.

For the second case, it is clearly that $v \in V_{t}$. Assume the list stored in $M[v]$ is $\left(v_{b_{1}}, d_{1}\right), \ldots,\left(v_{b_{m}}, d_{m}\right)$. Lemma 3 shows a lower bound for this case.

Lemma 3 Suppose the best possible candidate answer using such an $v\left(v \in M\right.$ and $\left.v \in V_{t}\right)$ as the answer root is $g_{2}$, then

$$
s\left(g_{2}\right)>\sum_{i=1}^{m} f\left(v_{b_{i}}\right) d_{i}+\left(1-f\left(v_{b_{i}}\right)\right) d\left(p_{i}\right),
$$

where $f\left(v_{b_{i}}\right)=1$ if $M[v]\left[b_{i}\right] \neq n i l$, and $f\left(v_{b_{i}}\right)=0$ otherwise. 
Notice that in Lemma 3 if $M[v]\left[b_{i}\right] \neq$ nil, then $d\left(p_{i}\right) \geq d_{i}$ due to the fact that $a_{i}$ is a min-heap. It follows $s\left(g_{2}\right) \leq s\left(g_{1}\right)$.

The termination condition. These $v$ 's represent all nodes that have not been fully explored. For case (i), we simply let $s\left(g_{1}\right)=\sum_{i=1}^{m} d\left(p_{i}\right)$; for case (ii), we find a vertex with the smallest possible $s\left(g_{2}\right)$ value w.r.t. the RHS of (2), and simply denote its best possible score as $s\left(g_{2}\right)$.

Denote the $k$ th smallest candidate answer identified in the algorithm as $g$, our search can safely terminate when $s(g) \leq \min \left(s\left(g_{1}\right), s\left(g_{2}\right)\right)=s\left(g_{2}\right)$. We denote this algorithm as BACKWARD. By Lemmas 1, 2, 3, we have Theorem 1.

Theorem 1 The BACKWARD method finds the top- $k$ answers $\mathcal{A}(q, k)$ for any top- $k$ keyword query $q$ on an RDF graph.

\section{Type-Based Summarization}

The BACKWARD method is clearly not scalable on large RDF graphs. For instance, the keyword "Armstrong" appears 269 times in our experimental DBpedia dataset, but only one is close to the keyword "Apollo 11", as in Figure 1. If we are interested in the smallest subgraphs that connect these two keywords, the BACKWARD method will initiate many random accesses to the data on disk, and has to construct numerous search paths in order to complete the search. However, the majority of them will not lead to any answers. Intuitively, we would like to reduce the input size to BACKWARD and apply BACKWARD only on the most promising subgraphs. We approach this problem by proposing a type-based summarization approach on the RDF data. The idea is that, by operating our keyword search initially on the summary (which is typically much smaller than the data), we can navigate and prune large portions of the graph that are irrelevant to the query, and only apply BACKWARD method on the smaller subgraphs that guarantee to find the optimal answers.

The intuition. The idea is to first induce partitions over the RDF graph $G$. Keywords being queried will be first concatenated by partitions. The challenge lies on how to safely prune connections (of partitions) that will not result in any top- $k$ answer. To this end, we need to calibrate the length of a path in the backward expansion that crosses a partition. However, maintaining the exact distance for every possible path is expensive, especially when the data is constantly changing. Therefore, we aim to distill an updatable summary from the distinct structures in the partitions such that any path length in backward expansion can be effectively estimated. The key observation is that neighborhoods in close proximity surrounding vertices of the same type often share similar structures in how they connect to vertices of other types.

Example 1. Consider the condensed view of Figure 1. The graph in Figure 6(a) is common for the 1-hop neighborhoods of $\mathrm{URI}_{3}$ and $\mathrm{URI}_{5}$ with the type SpaceMission.

This observation motivates us to study how to build a typebased summary for RDF graphs. A similar effort can be seen in [23], where a single schema is built for all the types of entities in the data. However, this is too restrictive as RDF data is known to be schemaless [10], e.g., entities of the same type do not have a unified property conformance.

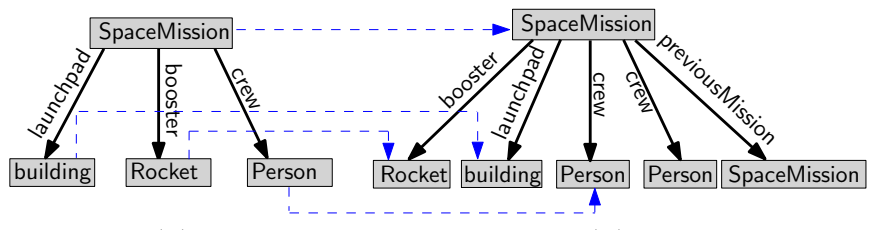

(a)

(b)

Fig. 6. Graph homomorphism across summaries.

\subsection{Outline and preliminaries}

Our approach starts by splitting the RDF graph into multiple, smaller partitions. Then, it defines a minimal set of common type-based structures that summarizes the partitions. Intuitively, the summary bookkeeps the distinct structures from all the partitions. In general, the keyword search can benefit from the summary in two perspectives. With the summary,

- we can obtain the upper and lower bounds for the distance traversed in any backward expansion without constructing the actual path (Section 6); and

- we can efficiently retrieve every partition from the data by collaboratively using SPARQL query and any RDF store without explicitly storing the partition (Section 15.

We first introduce two notions from graph theory: graph homomorphism and core.

Homomorphism across partitions. As in Figure 6(a), type vertices at close proximity are a good source to generate induced partitions of the data graph. However, if we were to look for such induced partitions that are exactly the same across the whole graph, we would get a large number of them. Consider another type-based structure in Figure 6(b), which is extracted from 1-hop neighbors around the vertex $\mathrm{URI}_{3}$ in Figure 1 Notice the two graphs are different, however Figure 6 a) is a subgraph of Figure 6 b). We consider discovering such embeddings between the induced partitions, so that one template can be reused to bookkeep multiple structures.

Definition 1 A graph homomorphism $f$ from a graph $G=$ $\{V, E\}$ to a graph $G^{\prime}=\left\{V^{\prime}, E^{\prime}\right\}$, written as $f: G \rightarrow G^{\prime}$, is a mapping function $f: V \rightarrow V^{\prime}$ such that (i) $f(x)=x$ indicates that $x$ and $f(x)$ have the same type; and (ii) $(u, v) \in E$ implies $(f(u), f(v)) \in E^{\prime}$ and they have the same label. When such an $f$ exists, we say $G$ is homomorphic to $G^{\prime}$.

Intuitively, embedding $G$ to $G^{\prime}$ not only reduces the number of structures we need to keep but also preserve any path from $G$ in $G^{\prime}$, as shown by Figure 6 (more expositions in Section 6). Finally, notice that homomorphism is transitive, i.e., $G \rightarrow G^{\prime}$ and $G^{\prime} \rightarrow G^{\prime \prime}$ imply that $G \rightarrow G^{\prime \prime}$.

Cores for individual partitions. A core is a graph that is only homomorphic to itself, but not to any one of its proper subgraphs (i.e., there is no homomorphism from a core to any of its proper subgraphs).

Definition $2 A$ core $c$ of a graph $G$ is a graph with the following properties: there exists a homomorphism from $c$ to $G$; there exists a homomorphism from $G$ to $c$; and $c$ is minimal (in the number of vertices) with these properties.

Intuitively, a core succinctly captures how different types of entities are connected, e.g., the partition in Figure 7(b) is converted to its core in Figure 77a) by eliminating one branch. 


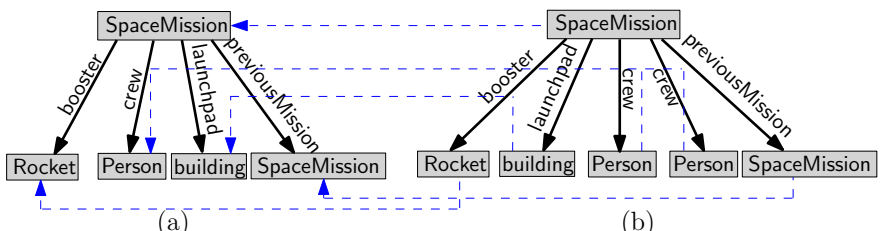

$(\bar{a})$

lemma summarizes the properties of our construction:

Lemma 4 Partitions in $\mathcal{P}$ are edge disjoint and the union of all partitions in $\mathcal{P}$ cover the entire graph $G$.

It is worth pointing out that Algorithm 2 takes an ad hoc order to partition the RDF data, i.e., visiting the set of entities from type 1 to type $n$ in order. A different order to partition the data could lead to different performance in evaluating a keyword query. However, finding an optimal order to partition the RDF data set is beyond the scope the paper, therefore we decide not to expand the discussion on this issue. graph into smaller but semantically similar and edge-disjoint subgraphs. Given our observation that nodes with the same type often share similar type-neighborhoods, we induce a distinct set of partitions for $G$ based on the types in $G$, using small subgraphs surrounding vertices of the same type. Our partitioning algorithm treats an input RDF dataset as a directed graph $G$ concerning only the type information, i.e., we use the condensed view of an RDF graph. For any vertex that does not have a type specified by the underlying dataset, we assign an universal type NA to them. Notice that graph partitioning is a well studied problem in the literature, here we do not propose any new technique in that respect but rather focus on how to build semantically similar partitions for our purpose. The partitioning algorithm is shown in Algorithm 2

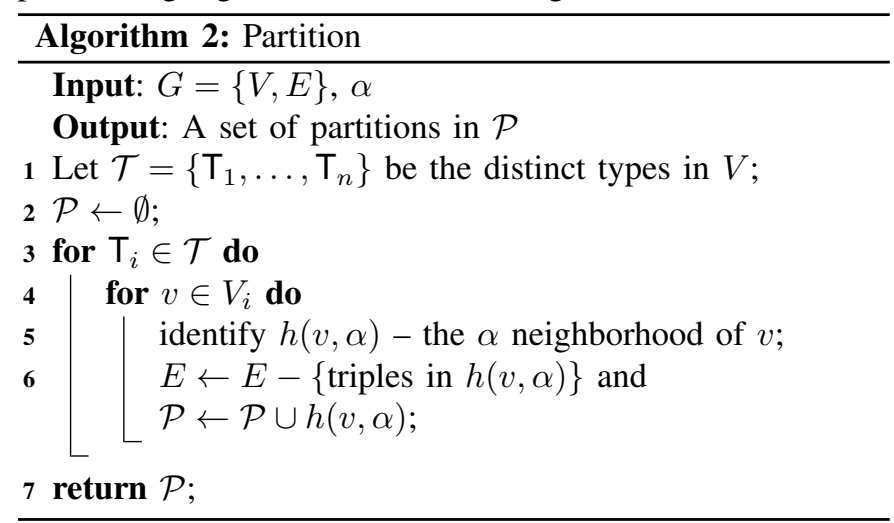

In Algorithm 2, suppose $G$ has $n$ distinct number of types $\left\{\mathrm{T}_{1}, \ldots, \mathrm{T}_{n}\right\}$, and we use the set $V_{i}$ to represent the vertices from $V$ that have a type $\mathrm{T}_{i}$ (line 4 ). We define the $\alpha$ neighborhood surrounding a vertex, where $\alpha$ is a parameter used to produce a set of edge disjoint partitions $\mathcal{P}$ over $G$. Formally, for any vertex $v \in V$ and a constant $\alpha$, the $\alpha$-neighborhood of $v$ is the subgraph from $G$ obtained by expanding $v$ with $\alpha$ hops in a breadth-first manner, denoted as $h(v, \alpha)$ (line 5), but subject to the constraint that the expansion only uses edges which have not been included by any partition in $\mathcal{P}$ yet. We define the $i$-hop neighboring nodes of $v$ as the set of vertices in $G$ that can be connected to $v$ through a directed path with exactly $i$ directed edges. Note that since we are using directed edges, it is possible the $i$-hop neighboring nodes of $v$ is an empty set. Clearly the nodes in $h(v, \alpha)$ are a subset of the $\alpha$-hop neighboring nodes of $v$ (since some may have already been included in another partition).

To produce a partition $\mathcal{P}$, we initialize $\mathcal{P}$ to be an empty set (line 2) and then iterate all distinct types (line 3). For a type $\mathrm{T}_{i}$ and for each vertex $v \in V_{i}$, we find its $\alpha$-neighborhood $h(v, \alpha)$ and add $h(v, \alpha)$ as a new partition into $\mathcal{P}$. The following

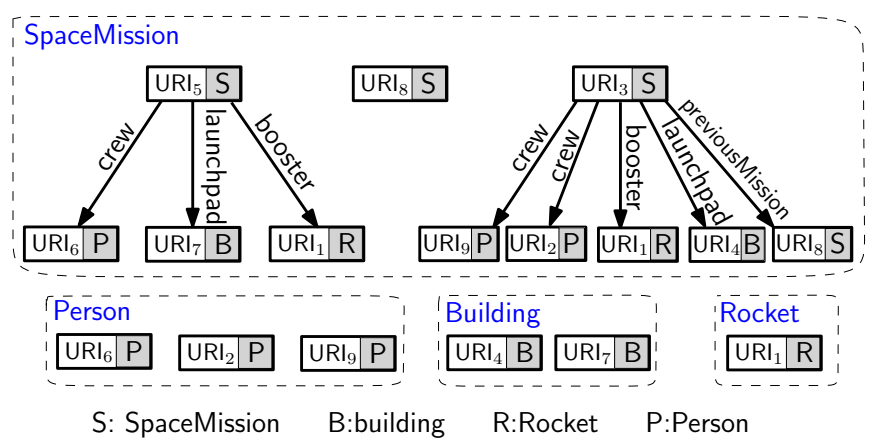

Fig. 8. Partitions $\mathcal{P}$ of the RDF data in Figure $1, \alpha=1$.

Note that the order in which we iterate through different types may affect the final partitions $\mathcal{P}$ we build. But no matter which order we choose, vertices in the same type always induce a set of partitions based on their $\alpha$-neighborhoods. For example, the partitions $\mathcal{P}$ of Figure 1 (as condensed in Figure 4 are always the ones shown in Figure 8, using $\alpha=1$.

\subsection{Summarization}

The intuition of summarization technique is as follows. The algorithm identifies a set of templates from the set of partitions $\mathcal{P}$. Such templates serve as a summary for the partitions. In addition, the summarization algorithm guarantees that every partition in $\mathcal{P}$ is homomorphic to one of the templates in the summary. As we will show in section 6 this property allows the query optimizer to (i) efficiently estimate any path length in the backward expansion without frequently accessing the RDF data being queried; and (ii) efficiently reconstruct the partitions of interest by querying the RDF data without explicitly storing and indexing the partitions.

We first outline our approach to summarize the distinct structures in a partition $\mathcal{P}$. Then, we discuss how to make it more practical by proposing our optimizations. Finally, we discuss the related indices in Section 5.4 The general framework of our approach is shown in Algorithm 3.

Given a partition $\mathcal{P}$, Algorithm 3 retrieves all the distinct structures and stores them in a set $S$. Algorithm 3 begins with processing partitions in $\mathcal{P}$ in a loop (line 2). For a partition $h_{i}$, we use its core $c$ to succinctly represent the connections between different types in $h_{i}$ (line 3 ). Once a core $c$ is constructed for a partition, we scan the existing summary structures in $S$ to check (a) if $c$ is homomorphic to any existing structure $s_{i}$ in $S$; or (b) if any existing structure $s_{i}$ in $S$ is homomorphic to $c$. In the former case, we terminate the scan and $S$ remains intact (without adding $c$ ), as in lines 5-6; in 
the latter case, we remove $s_{i}$ from $S$ and continue the scan, as in lines 7-8. When $S$ is empty or $c$ is not homomorphic to any of the structures in $S$ after a complete scan on $S$, we add $c$ into $S$. We repeat the procedure for all partitions in $\mathcal{P}$.

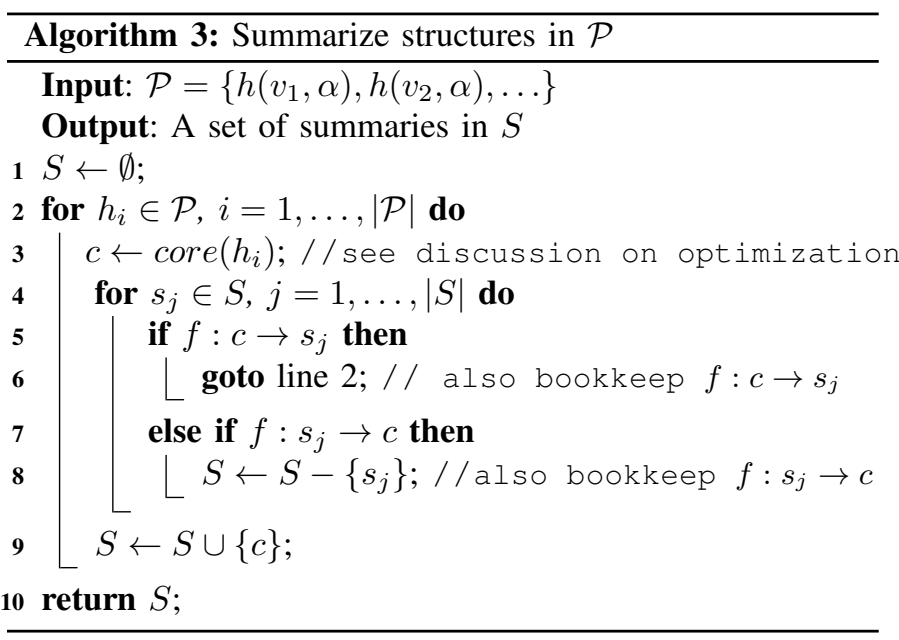

Improving efficiency and reducing $|S|$. There are two practical problems in Algorithm 3 . First, the algorithm requires testing subgraph isomorphism for two graphs in lines 3, 5 and 7, which is NP-hard. Second, we want to reduce $|S|$ as much as possible so that it can be cached in memory for query processing. The latter point is particularly important for RDF datasets that are known to be irregular, e.g., DBpedia.

The optimization is as follows. Before line 3 of Algorithm 3 consider each partition $h(v, \alpha)$ in $\mathcal{P}$, which visits the $\alpha$ neighborhood of $v$ in a breadth-first manner. We redo this traversal on $h(v, \alpha)$ and construct a covering tree for the edges in $h(v, \alpha)$, denoted as $h_{t}(v, \alpha)$. In more detail, for each visited vertex in $h(v, \alpha)$, we extract its type and create a new node in $h_{t}(v, \alpha)$ (even if a node for this type already exists). By doing so, we build a tree $h_{t}(v, \alpha)$ which represents all the distinct type-paths in $h(v, \alpha)$. In the rest of the algorithm (lines 3-10), we simply replace $h(v, \alpha)$ with $h_{t}(v, \alpha)$.

Example 2. As in Figure 9, a tree $h_{t}\left(v_{1}, 2\right)$ is built for the partition $h\left(v_{1}, 2\right)$. Notice that the vertex $v_{4}$ is visited three times in the traversal (across three different paths), leading to three distinct nodes with type $\mathrm{T}_{4}$ created in $h_{t}\left(v_{1}, 2\right)$. In the same figure, a tree $h_{t}\left(v_{5}, 2\right)$ is built from the partition $h\left(v_{5}, 2\right)$ and isomorphic to $h_{t}\left(v_{1}, 2\right)$.

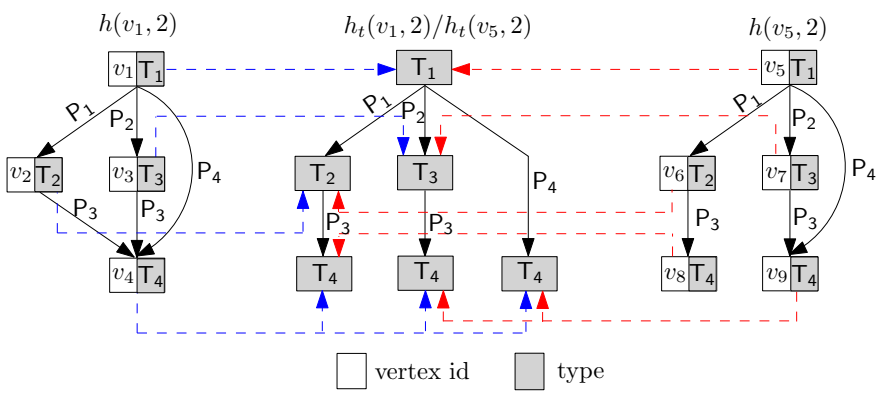

Fig. 9. A tree structure for two partitions.

There are two motivations behind this move. First, using the covering tree instead of the exact partition potentially reduces the size of the summary $S$. As seen in Figure 9 . two partitions with distinct structures at the data level (e.g., $h\left(v_{1}, 2\right)$ and $\left.h\left(v_{5}, 2\right)\right)$ could share an identical structure at the type level. Taking advantage of such overlaps is the easiest way to reduce the number of distinct structures in $S$. The second reason is efficiency. Whereas testing subgraph isomorphism is computationally hard for generic graphs, there are polynomial time solutions if we can restrict the testing on trees [21] leading to better efficiency. For instance, to find the core of a covering tree $h_{t}$, it simply amounts to a bottom-up and recursive procedure to merge the homomorphic branches under the same parent node in the tree.

\subsection{Auxiliary indexing structures}

To facilitate the keyword search, along with the summary $S$, we maintain three auxiliary (inverted) indexes.

A portal node $\ell$ is a data node that is included in more than one partitions (remember that partitions are edge-disjoint, not node disjoint). Intuitively, a portal node joins different partitions. A partition may have multiple portals but usually much less than the total number of nodes in the partition. Portal nodes allow us to concatenate different partitions. In the first index, dabbed portal index, for each partition $h(v, \alpha)$, we assign it a unique id, and associate it with the list of portals in the partition. In practice, since the partition root node $v$ is unique in each partition, we can simply use it to denote the partition $h(v, \alpha)$ when the context is clear.

Recall that we use $h_{t}(v, \alpha)$ to represent $h(v, \alpha)$, where a vertex in $h(v, \alpha)$ could correspond to more than one vertex in $h_{t}(v, \alpha) . \sigma\left(v_{i}\right)$ registers the fact that there are more than one $v_{i}$ in $h_{t}(v, \alpha)$ and $\sigma\left(v_{i}\right)$ also denotes the set of all $v_{i} \mathrm{~s}$ in $h_{t}(v, \alpha)$. Without loss of generality, let $\Sigma=\left\{\sigma\left(v_{1}\right), \sigma\left(v_{2}\right), \ldots\right\}$ denote all the one-to-many mappings in a partition. For instance, consider $h\left(v_{1}, 2\right)$ and $h_{t}\left(v_{1}, 2\right)$ in Figure 9, $\Sigma \leftarrow\left\{\sigma\left(v_{4}\right)=\right.$ $\left.\left\{T_{4}\right\}\right\}$. The second index, dabbed partition index, is to map the partition root $v$ of $h(v, \alpha)$ to its $\Sigma$. Intuitively, this index helps rebuild from $h_{t}(v, \alpha)$ a graph structure that is similar to $h(v, \alpha)$ (more rigorous discussion in section 6).

The third index, dabbed summary index, maps data nodes in partitions to summary nodes in $S$. In particular, we assign a unique id sid to each summary in $S$ and denote each node in $S$ with a unique id nid. For any node $u$ in a partition $h(v, \alpha)$, this index maps the node $u$ to an entry that stores the partition root $v$, the id sid of the summary and the id nid of the summary node that $u$ corresponds to. Notice that since $h_{t}(v, \alpha)$ is built in a breadth-first traversal, we can easily compute the shortest path from $v$ to any node in $h_{t}(v, \alpha)$ using this index.

To obtain the homomorphic mappings from each $h_{t}(v, \alpha)$ to a summary in $S$, one needs to maintain a $\log$ for all the homomorphisms found during the construction of $S$, as in lines 6 and 8. Once $S$ is finalized, we trace the mappings in this $\log$ to find the mappings from data to summaries. As each partition (represented by its core) is either in the final $S$ or is homomorphic to one other partition, the size of the log is linear to the size of $G$. An example for the log is in Figure $10\left(h_{t}^{i}\right.$ is the covering tree for the $i$-th partition). It shows sets of trees (and their homomorphic mappings); each set is associated with a summary in $S$, that all trees in that set are homomorphic to. To find the final mappings, we scan each set of trees in the 
$\log$ and map the homomorphisms of each entry in a set to the corresponding entry in $S$, i.e., the blue arrows in Figure 10. We remove the log once all the mappings to $S$ are found.

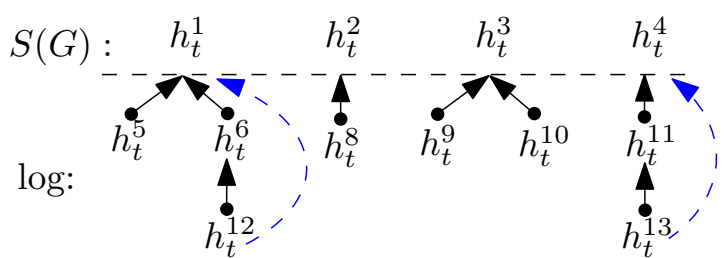

Fig. 10. All the homomorphism in building $S$.

\section{KEYWORD SEARCH WITH SUMMARY}

Next, we present a scalable and exact search algorithm. It performs a two-level backward search: one backward search at the summary-level, and one at the data-level. Only for identified connected partitions that are found to contain all the distinct keywords at the summary-level and whose score could enter the top- $k$ answers, do we initiate a backward search at the data-level on the selected partitions. Remember that path-length computation is at the heart of backward search and pruning. While working at the summary-level, exact path lengths are not available. Therefore, we first show how to estimate the path length of the actual data represented by our summary. Then, we proceed to describe the algorithm in detail.

\subsection{Bound the shortest path length}

At the summary-level, any shortest path in the underlying RDF graph must go through a number of partitions, and for each intermediate partition the path connects two of its portals, i.e., an entrance and an exit node. By construction, the shortest distance from the partition root $v$ of a partition to any vertex $u$ in the same partition can be computed with the summary index. By triangle inequality, the shortest distance $d\left(v_{1}, v_{2}\right)$ for any two vertices $v_{1}$ and $v_{2}$ in a partition with a partition root $v$ can be upper bounded by $d\left(v_{1}, v_{2}\right) \leq d\left(v, v_{1}\right)+d\left(v, v_{2}\right)$, and lower bounded by $d\left(v_{1}, v_{2}\right) \geq\left|d\left(v, v_{1}\right)-d\left(v, v_{2}\right)\right|$. Yet, a possibly tighter lower bound can be found by using the correspondent summary of the partition that is rooted at $v$ and Lemma 5.

Lemma 5 Given two graphs $g$ and $h$, if $f: g \rightarrow h$, then $\forall v_{1}$, $v_{2} \in g$ and their homomorphic mappings $f\left(v_{1}\right), f\left(v_{2}\right) \in h$, $d\left(v_{1}, v_{2}\right) \geq d\left(f\left(v_{1}\right), f\left(v_{2}\right)\right)$.

The homomorphic mappings between a partition $h$, its covering tree $h_{t}$, and its summary $s$ in $S$ are shown in Figure 11(a). Notice that due to the optimization we employ in Section 5.3, there is no homomorphism from $h$ to $s$, so that we can not apply Lemma 5 directly. To obtain a lower bound for the distance of any two vertices in $h$, we need to rebuild a homomorphic structure for $h$ from its summary $s$ and $h_{t}$.

To do so, we first define a mapping function Join, which takes as input a graph $g$, a list of disjoint sets of vertexes $\left\{V_{t_{1}}^{\prime}, V_{t_{2}}^{\prime}, \ldots\right\}$ and outputs a new graph $g^{\prime}$, written as $g^{\prime}=\operatorname{Join}\left(g(V, E),\left\{V_{t_{1}}^{\prime}, V_{t_{2}}^{\prime}, \ldots\right\}\right)$. In particular, $V_{t_{i}}^{\prime} \subseteq V$ and vertexes in $V_{t_{i}}^{\prime}$ are all of type $t_{i}$. The function Join constructs $g^{\prime}$ as follows:

1. Initialize $g^{\prime}$ as $g$;

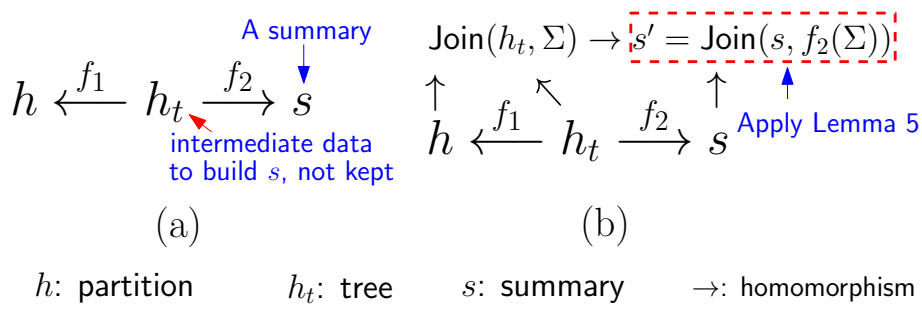

Fig. 11. Homomorphic mappings

2. For the vertexes in $V_{t_{i}}^{\prime}$ of $g^{\prime}$, merge them into a single node $v_{i}^{\prime}$ of type $t_{i}$, and all edges incident to the vertexes in $V_{t_{i}}^{\prime}$ are now incident to $v_{i}^{\prime}$;

3. Repeat step 2 for all $i$ s.

Notice that the function Join itself is a homomorphic mapping, which constructs a homomorphism from $g$ to $g^{\prime}$. Also recall $\sigma(x)$ of a partition $h$ registers the fact that a type node $x$ in $h$ has more than one replicas in $h_{t}$ and hence $\sigma(x)$ is a one-tomany mapping from $x$ in $h$ to the set of all vertexes of type $x$ in $h_{t}$ and $\Sigma$ of $h_{t}$ is the set of all such mappings.

Example 3. Use the examples in Figure 9] for illustration. Join $\left(h_{t}\left(v_{1}, 2\right),\left\{\sigma\left(\mathrm{T}_{4}\right)\right\}\right)$ rebuilds $h\left(v_{1}, 2\right)$ in Figure 9 and hence there is a homomorphism from $h\left(v_{1}, 2\right)$ to $\operatorname{Join}\left(h_{t}\left(v_{1}, 2\right),\left\{\sigma\left(\mathrm{T}_{4}\right)\right\}\right)$, i.e., isomorphism. On the other hand, Join $\left(h_{t}\left(v_{5}, 2\right),\left\{\sigma\left(\mathrm{T}_{4}\right)\right\}\right)$ equals $h\left(v_{1}, 2\right)$. Although it does not reconstruct $h\left(v_{5}, 2\right)$ this time, the Join function in this case still produces a homomorphic mapping from $h\left(v_{5}, 2\right)$ to Join $\left(h_{t}\left(v_{5}, 2\right),\left\{\sigma\left(\mathrm{T}_{4}\right)\right\}\right)$ since it is not hard to see that $h\left(v_{5}, 2\right)$ is homomorphic to $h\left(v_{1}, 2\right)$ in Figure 9 .

More formally, we have:

Lemma 6 For a partition $h$ and its covering tree $h_{t}$, there is a homomorphism from $h$ to Join $\left(h_{t}, \Sigma\right)$.

In what follows, we show how to build a homomorphism from Join $\left(h_{t}, \Sigma\right)$ to a graph $s^{\prime}$ derived from the summary $s$ of $h_{t}$. With this and by the transitivity of homomorphism and Lemma 6, it follows $h$ is homomorphic to $s^{\prime}$ and hence Lemma 5 can be applied (as shown in Figure 11 b)).

By Algorithm 2, every $h_{t}$ from a partition $h$ is homomorphic to a summary $s$ in $S$ (see the relations in Figure 11 a)). Assume the homomorphism is $f_{2}: h_{t} \rightarrow s$. Given the $\Sigma$ of a partition $h$, define $f_{2}(\Sigma)=\left\{f_{2}(\sigma(v)) \mid \sigma(v) \in \Sigma\right\}$ where $f_{2}(\sigma(v))=\left\{f_{2}(u) \mid u \in \sigma(v) \wedge u \in h_{t} \wedge f_{2}(u) \in s\right\}$, i.e., the set of the mapped vertexes of $\sigma(v)$ in $s$ by the homomorphism $f_{2}$. Further, we have the following result:

Lemma 7 For a partition $h$, its covering tree $h_{t}$ and its summary $s$ that has $f_{2}: h_{t} \rightarrow s$, there is a homomorphism from Join $\left(h_{t}, \Sigma\right)$ to Join $\left(s, f_{2}(\Sigma)\right)$.

By Lemmas 6, 7 and the transitivity of homomorphism, a partition $h$ is homomorphic to Join $\left(s, f_{2}(\Sigma)\right)$, as shown in Figure 11 (b). Notice $f_{2}$ is a part of our summary index, which maps a vertex in data to a vertex in summary. Finally, given any two vertices in a partition $h$, their shortest distance can be (lower) bounded by combining Lemmas 5, 6, 7, and using any shortest path algorithm, e.g., Dijkstra's algorithm, to find the shortest distance between the correspondent mappings on $\operatorname{Join}\left(s, f_{2}(\Sigma)\right)$. In practice, we use the larger lower bound from either the summary or the triangle inequality. 


\subsection{The algorithm}

The algorithm is in Algorithm 4, dabbed the SUMm method.

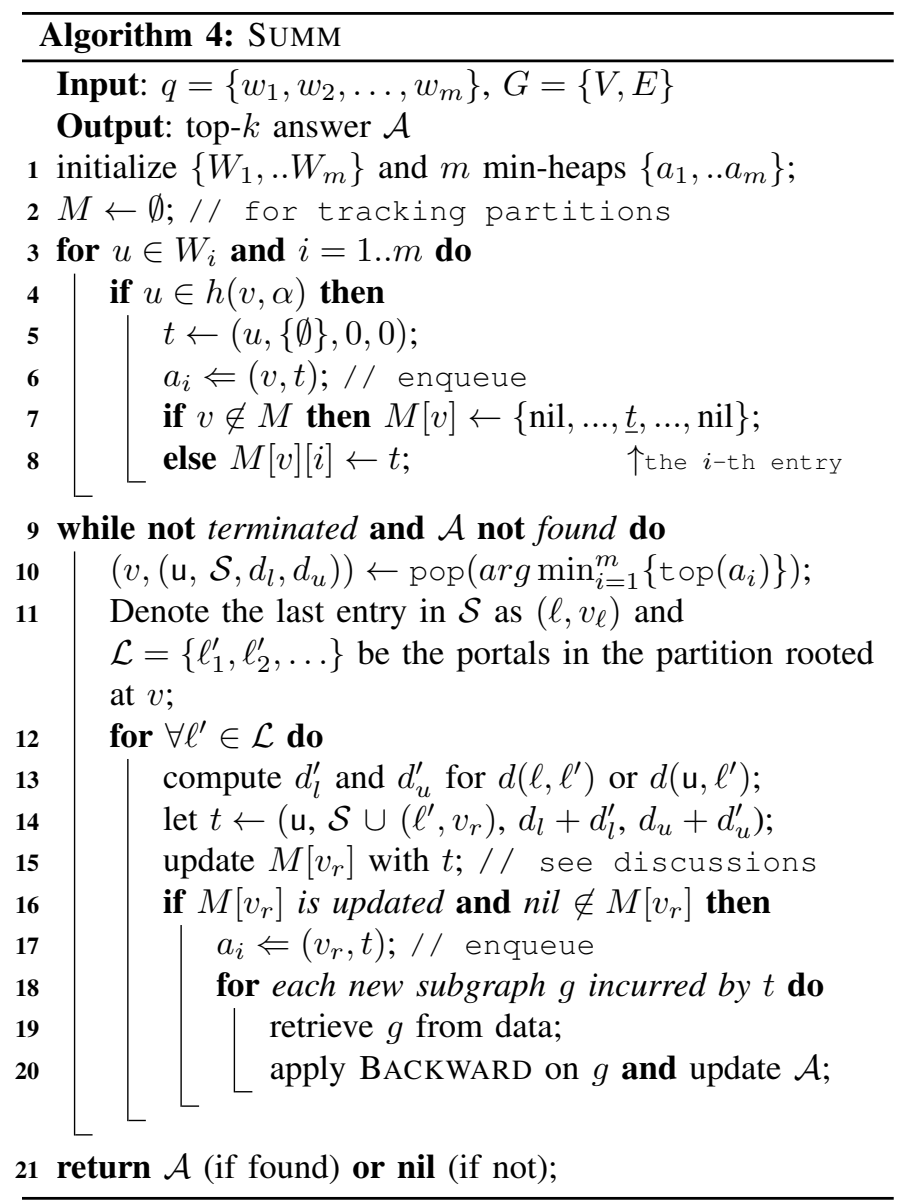

Data structures. Like the BACKWARD method in section 4. we define $\left\{W_{1}, W_{2}, \ldots, W_{m}\right\}$, where $W_{i}$ is the set of vertexes containing the keyword $w_{i}$. We also initialize $m$ priority queues $\left\{a_{1}, \ldots, a_{m}\right\}$ and maintain a set $M$ of entries, one for each visited partition. Each entry in $M$ stores a unique partition root followed by $m$ lists. The $i$-th list records all the reachable vertexes containing keyword $w_{i}$ and through which other partitions they connect to the current partition in the search. An entry of $M$ is in the form of quadruples - each can be represented as $\left(\mathrm{u}, \mathcal{S}, d_{l}, d_{u}\right)$. Here, the node $\mathrm{u}$ is the first vertex in the backward expansion and contains the keyword $w_{i}$. The expansion reaches the current partition by routing through a sequence of the portals from some partitions, stored in $\mathcal{S}$ as a sequence of (portal, partition root) pairs. A sequence $\mathcal{S}$ defines a path (of partitions) that starts at $\mathrm{u}$. Denote the lower and upper bounds for the path in $\mathcal{S}$ as $d_{l}$ and $d_{u}$.

Example 4. A sub-sequence $\left\{\left(\ell, v_{a}\right),\left(\ell^{\prime}, v_{b}\right)\right\}$ of $\mathcal{S}$ indicates that the path first enters the partition rooted at $v_{a}$ and exits the partition from one of its portals $\ell$. From the portal $\ell$, this path then enters a partition rooted at $v_{b}$ and leaves the partition from the portal $\ell^{\prime}$. We are interested in (lower and upper) bounding the shortest distance that connects two adjacent portals in $\mathcal{S}$, e.g., $d\left(\ell, \ell^{\prime}\right)$ in the partition rooted at $v_{b}$.

Example 5. In Figure 12, assume $m=2$ (i.e., the query has two keywords) and an entry in $M$ for a partition rooted at $v$ is shown as below. The entry records that there is a path (of

\begin{tabular}{cc}
\hline$w_{1}$ & $w_{2}$ \\
\hline$t_{1}=\left(v_{a},\left\{\left(\ell_{2}, v_{0}\right)\right\}, 5,7\right)$ & $t_{2}=\left(v_{b},\left\{\left(\ell_{1}, v_{4}\right),\left(\ell_{0}, v_{5}\right)\right\}, 3,5\right)$ \\
& $t_{3}=\left(v_{c},\left\{\left(\ell_{3}, v_{2}\right)\right\}, 5,6\right)$ \\
\hline
\end{tabular}

Fig. 12. An entry in $M$ for the partition rooted at $v$

partitions) from $w_{1}$ that reaches the current partition rooted at $v$. This path starts at $v_{a}$, enters the concerned partition from portal $\ell_{2}$ and has a length of at least 5 hops and at most 7 hops. To reach the partition rooted at $v$, the path has already passed through a partition rooted at $v_{0}$. Same for $w_{2}$, the concerned partition is reachable from two paths starting at $v_{b}$ and $v_{c}$ respectively, both contain the keyword $w_{2}$.

The algorithm. With the data structures in place, the algorithm proceeds in iterations.

- In the first iteration. For each vertex u from $W_{i}$, we retrieve the partition root $v$ that $\mathrm{u}$ corresponds to, from the summary index. Next, if there is an entry for $v$ in $M$, we append a quadruple $t=(\mathrm{u},\{\emptyset\}, 0,0)$ to the $i$-th list of the entry; otherwise we initialize a new entry for $v$ in $M$ (with $m$ empty lists) and update the $i$-th list with $t$, as in lines 7-8. We also add an entry $(v, t)$ to the priority queue $a_{i}$ (entries in the priority queue are sorted in ascending order by their lower bound distances in $t$ 's). We repeat this process for all $W_{i}$ 's for $i=1, \ldots, m$, which completes the first iteration (lines 3-8).

- In the $j$-th iteration. We pop the smallest entry from all $a_{i}$ 's, say $\left(v,\left(\mathrm{u}, \mathcal{S}, d_{l}, d_{u}\right)\right)$ (line 10). We denote the partition rooted at $v$ as the current partition. Denote the last pair in $\mathcal{S}$ as $\left(\ell, v_{\ell}\right)$, which indicates that the path leaves the partition rooted at $v_{\ell}$ and enters the current partition using portal $\ell$. Next, for the current partition, we find its portals $\mathcal{L}=\left\{\ell_{1}^{\prime}, \ell_{2}^{\prime}, \ldots\right\}$ from the portal index. For each $\ell^{\prime}$ in $\mathcal{L}$, we compute the lower and upper bounds for $d\left(\ell, \ell^{\prime}\right)$ (or $d\left(\mathrm{u}, \ell^{\prime}\right)$ if $\ell=n i l$ ) in the current partition using the approach discussed in section 6.1, denoted as $d_{l}^{\prime}$ and $d_{u}^{\prime}$ (line 13). A portal $\ell^{\prime}$ can connect the current partition to a set $P^{\prime}$ of neighboring partitions. For each partition in $P^{\prime}$, denoted by its partition root $v_{r}$, we construct a quadruple $t=(\mathrm{u}$, $\left.\mathcal{S} \cup\left(\ell^{\prime}, v_{r}\right), d_{l}+d_{l}^{\prime}, d_{u}+d_{u}^{\prime}\right)$ as in line 14. We also search for the entry of $v_{r}$ in $M$ and update its $i$-th list with $t$ in the same way as in the first iteration. However, if either of the following cases is satisfied, we stop updating the entry for $v_{r}$ in $M$ : (i) adding $\ell^{\prime}$ to $\mathcal{S}$ generates a cycle; and (ii) $d_{l}+d_{l}^{\prime}$ is greater than the $k$-th largest upper bound in the $i$-th list. Otherwise, we also push $\left(v_{r}, t\right)$ to the queue $a_{i}$.

At any iteration, if a new quadruple $t$ has been appended to the $i$-th list of an entry indexed by $v$ in $M$, and all of its other $m-1$ lists are non-empty, then the partition rooted at $v$ contains potential answer roots for the keyword query. To connect the partitions containing all the keywords being queried, we find all the possible combinations of the quadruples from the $(m-1)$ lists, and combine them with $t$. Each combination of the $m$ quadruples denotes a connected subgraph having all the keywords being queried.

Example 6. In Figure 12, denote the new quadruple just inserted to the first list of an entry in $M$ as $t_{1}$. Since both of its lists are now non-empty, two combinations can be found, i.e., $\left(t_{1}, t_{2}\right)$ and $\left(t_{1}, t_{3}\right)$, which leads to two conjunctive subgraphs. Using the partition information in the quadruples, we can 
easily locate the correspondent partitions.

We study how to access the instance data for a partition in Section 7. Once the instance data from the selected partitions are ready, we proceed to the second-level backward search by applying the BACKWARD method to find the top- $k$ answers on the subgraph concatenated by these partitions (line 20). In any phase of the algorithm, we track the top- $k$ answers discovered in a priority queue.

- Termination condition. The following Lemmas provide a correct termination condition for the SUMM method.

Lemma 8 Denote an entry in the priority queue as $(v,(u, \mathcal{S}$, $\left.\left.d_{l}, d_{u}\right)\right)$, then for any $v^{\prime}$ in the partition rooted at $v$ and the length of any path starting from $u$ and using the portals in $\mathcal{S}$ is $d\left(u, v^{\prime}\right) \geq d_{l}$.

Lemma 9 Denote the top entry in the priority queue $a_{i}$ as $\left(v,\left(u, \mathcal{S}, d_{l}, d_{u}\right)\right)$, then for any explored path $p$ from $w_{i}$ in the queue $a_{i}$, the length of $p$, written as $d(p)$, has $d(p) \geq d_{l}$.

We denote the set of all unexplored partitions in $\mathcal{P}$ as $\mathcal{P}_{t}$. For a partition $h$ rooted at $v$ that has not been included in $M$, clearly, $h \in \mathcal{P}_{t}$. The best possible score for an answer root in $h$ is to sum the $d_{l}$ 's from all the top entries of the $m$ expansion queues, i.e., $a_{1}, \ldots, a_{m}$. Denote these $m$ top entries as $\left(v_{1},\left(u_{1}\right.\right.$, $\left.\left.\mathcal{S}^{1}, d_{l}^{1}, d_{u}^{1}\right)\right), \ldots,\left(v_{m},\left(u_{m}, \mathcal{S}^{m}, d_{l}^{m}, d_{u}^{m}\right)\right)$, respectively. Then,

Lemma 10 Let $g_{1}$ be a possible unexplored candidate answer rooted at a vertex in a partition $h$, with $h \in \mathcal{P}_{t}$,

$$
s\left(g_{1}\right)>\sum_{i=1}^{m} d_{l}^{i} .
$$

Next, consider the set of partitions that have been included in $M$, i.e., the set $\mathcal{P}-\mathcal{P}_{t}$. For a partition $h \in \mathcal{P}-\mathcal{P}_{t}$, let the first quadruple from each of the $m$ lists for its entry in $M$ be: $t_{1}=\left(\hat{u}_{1}, \hat{\mathcal{S}}_{1}, \hat{d}_{l}^{1}, \hat{d}_{u}^{1}\right), \ldots, t_{m}=\left(\hat{u}_{m}, \hat{\mathcal{S}_{m}}, \hat{d}_{l}^{m}, \hat{d}_{u}^{m}\right)$ (note that due to the order of insertion, each list has been implicitly sorted by the lower bound distance $\hat{d}_{l}$ in ascending order), where $t_{j}=n i l$ if the $j$-th list is empty. Then, we have:

Lemma 11 Denote the best possible unexplored candidate answer as $g_{2}$, which is rooted at a vertex in the partition $h$, where $h \in \mathcal{P}-\mathcal{P}_{t}$, then

$$
s\left(g_{2}\right)>\sum_{i=1}^{m} f\left(t_{i}\right) \hat{d}_{l}^{i}+\left(1-f\left(t_{i}\right)\right) d_{l}^{i},
$$

where $f\left(t_{i}\right)=1$ if $t_{i} \neq$ nil otherwise $f\left(t_{i}\right)=0$.

Finally, we can derive the termination condition for the search.

The termination condition. We denote the score of the best possible answer in an unexplored partition as $s\left(g_{1}\right)$, as defined by the RHS of (3); and the score of the best possible answer in all explored partitions as $s\left(g_{2}\right)$, as defined by the RHS of (4). Denote the candidate answer with the $k$-th smallest score during any phase of the algorithm as $g$. Then, the backward expansion on the summary level can safely terminate when $s(g) \leq \min \left(s\left(g_{1}\right), s\left(g_{2}\right)\right)$. By Lemmas 10 and 11 , we have:

Theorem 2 SuMM finds the top- $k$ answers $\mathcal{A}(q, k)$ for any top- $k$ keyword search query $q$ on an RDF graph.

Sections 13 and 14 in the online appendix discuss the complexity of SUMM and further elaborate its correctness.

\section{ACCESSING DATA AND UPDATE}

The SUMM algorithm uses the summary of the RDF data to reduce the amount of data accessed in the BACKWARD method. For the algorithm to be effective, we should be able to efficiently identify and retrieve the instance data from selected partitions. One option is to store the triples by partitions and index on their partition ids, i.e., adding another index to the algorithm. But then whenever an update on the partition happens, we need to update the index. Furthermore, the approach enforces a storage organization that is particular to our methods (i.e., not general). In what follows, we propose an alternative efficient approach that has no update overhead and requires no special storage organization. Our approach stores the RDF data in an RDF store and works by dynamically identifying the data of a partition using appropriately constructed SPARQL queries that retrieve only the data for that partition.

Since graph homomorphism is a special case of homomorphism on relational structure (i.e., binary relations) [16] and the fact that relational algebra [20] is the foundation of SPARQL, we can use the Homomorphism Theorem [1] to characterize the results of two conjunctive SPARQL queries.

Theorem 3 Homomorphism Theorem [1]. Let $q$ and $q^{\prime}$ be relational queries over the same data $D$. Then $q^{\prime}(D) \subseteq q(D)$ iff there exists a homomorphism mapping $f: q \rightarrow q^{\prime}$.

Recall that $f_{1}: h_{t} \rightarrow h$ (see Figure 11(a)) and for each $h_{t}$, we extract a core $c$ from $h_{t}$. By definition, $c$ is homomorphic to $h_{t}$, thus $c$ is homomorphic to $h$ (transitivity). Using $c$ as a SPARQL query pattern can extract $h$ due to Theorem 3 .

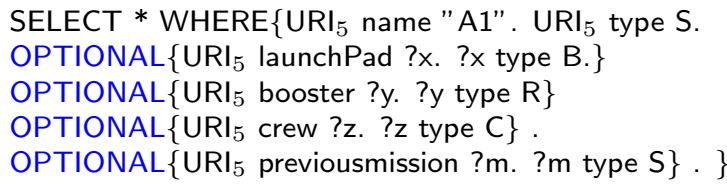

Fig. 13. A query to retrieve the targeted partition.

There are two practical issues the need our attention. First, there is usually a many-to-one mapping from a set of $h_{t}$ 's to the same core $c$ - leading to a low selectivity by using $c$ as the query pattern. To address this issue, we can bind constants from the targeted partition to the respective variables in query pattern. These constants include the root and the portals of the targeted partition which are retrievable from the indexes. The second issue is that in our construction of $S$, we do not explicitly keep every $c$. Instead, a core $c$ is embedded (by homomorphism) to a summary $s \in S$, where $c$ is a subtree of $s$. To construct a SPARQL query from $s$, we first need to find a mapping for the partition root in $s$, then the triple patterns corresponding to the subtree in $s$ are expressed in (nested) OPTIONALS from the root to the leaves. For example, the SPARQL query for the partition rooted at $\mathrm{URI}_{5}$ in Figure 8 can be constructed by using the summary in Figure 7(a). Notice that $\mathrm{URI}_{5}$ is bound to the root to increase selectivity.

Our approach also supports efficient updates, which is addressed in Section 15 in the online appendix. 


\section{EXPERIMENTS}

We implemented the BACKWARD and SUMM methods in $\mathrm{C}++$. We also implemented two existing approaches proposed in [23] and [14]. We denote them as SCHEMA and BLINKS respectively. All experiments were conducted on a 64-bit Linux machine with $6 \mathrm{~GB}$ of memory.

\begin{tabular}{ccccc}
\hline \hline LUBM & Wordnet & Barton & BSBM & DBpedia Infobox \\
\hline 14 & 15 & 30 & 1520 & 5199 \\
\hline
\end{tabular}

Fig. 14. Number of distinct types in the datasets

\begin{tabular}{ccccc}
\hline \hline LUBM & Wordnet & Barton & BSBM & DBpedia Infobox \\
\hline 5 & 2 & 40 & 70 & 30 \\
\hline
\end{tabular}

Fig. 15. Number of triples in the datasets $\left(\times 10^{6}\right)$

Datasets: We used large sythetic and real RDF data sets for experiments. Sythetic data sets are generated by popular RDF benchmarks. The first is the Lehigh University Benchmark (LUBM) [13], which models universities with students, departments, etc. With its generator, we created a default dataset of 5 million triples and varied its size up to 28 million triples. The second is the Berlin SPARQL Benchmark (BSBM) [5], which models relationships of products and their reviews. Unlike LUBM where data are generated from a fixed set of templates, BSBM provides interfaces to scale the data on both size and complexity of its structure. In particular, it provides means for adding new types in the data. The rest of the data sets are popular real RDF data sets, i.e., Wordnet, Barton and DBpedia Infobox. The number of distinct types for the data sets are shown in Figure 14 and their sizes are reported in Figure 15. Notice DBpedia and BSBM are very irregular in structure, i.e., both have more than 1,000 distinct types. BSBM is also large in size (70 million triples by default).

Implementation and setup: We used the disk-based $\mathrm{B}^{+}$-tree implementation from the TPIE library to build a Hexstorelike [24] index on the RDF datasets. For subgraph isomorphism test, we used the VFLib. We assume each entity in the data has one type. For an entity that has multiple types, we bind the entity to its most popular type. To store and query RDF data with SPARQL, we use Sesame [6]. In all experiments, if not otherwise noted, we built the summary for 3-hop neighbors, i.e., $\alpha=3$, and set $k=5$ for top- $k$ queries.

\subsection{Evaluating summarization algorithms}

Time on the summarization process. We start with a set of experiments to report the time (in log scale) in building a summary. For LUBM, we vary the size of the data from 100 thousand triples to 28 million triples. In Figure 16(a), we plot the total time for building the summary, which includes: the time spent to find homomorphic mappings (i.e., performing subgraph isomorphism tests) and the time spent for the rest of operations, e.g., partitioning the graph, and constructing the inverted indexes. The latter cost dominates the summarization process for all the cases in LUBM datasets. The same trend can also be observed in all the other datasets, as shown in Figure 16(b). Notice that the summary used by SuMM is built once and thereafter incrementally updatable whenever the data get updated. For comparison, we study

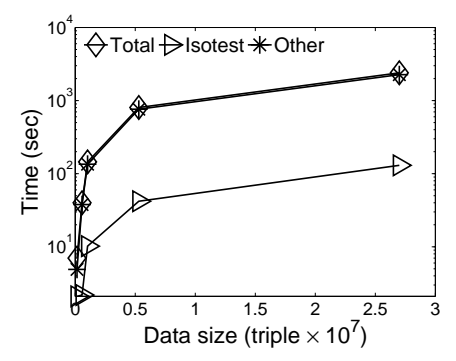

(a) LUBM.

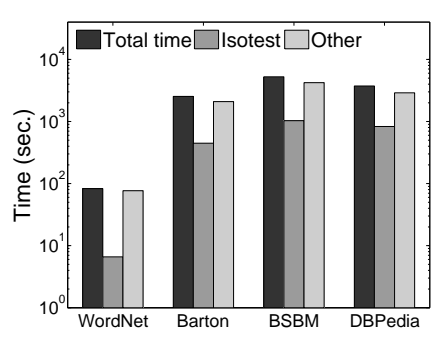

(b) Other datasets.
Fig. 16. Time for the summary construction.

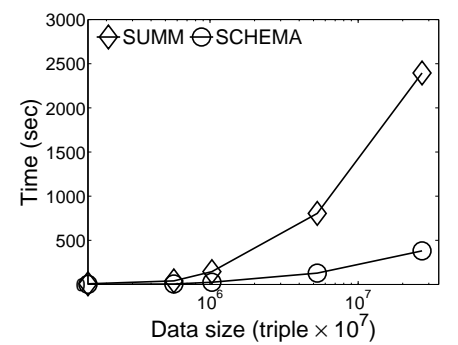

(a) LUBM.

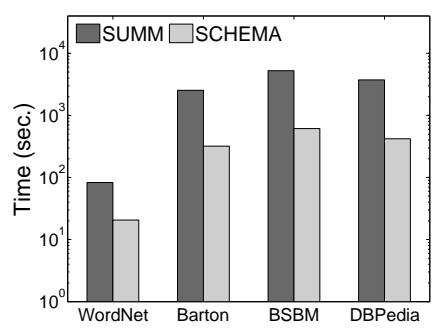

(b) Other datasets.
Fig. 17. Time for the summarization: Summ vs. SCHEMA.

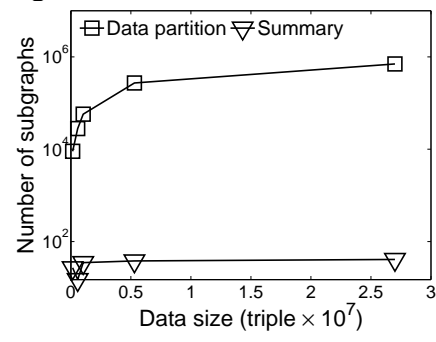

(a) LUBM.

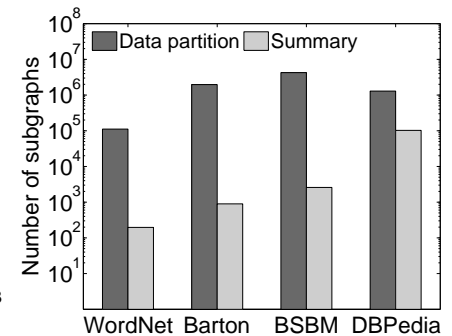

(b) Other datasets.
Fig. 18. \# subgraphs: partitions vs. summary.

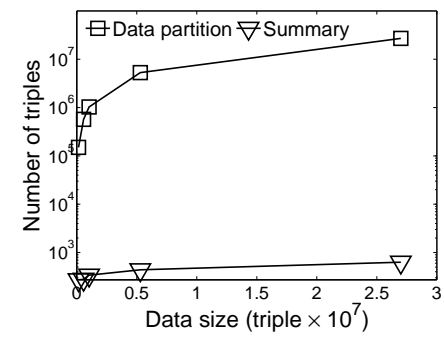

(a) LUBM.

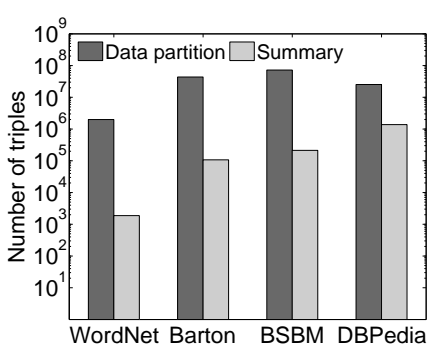

(b) Other datasets.
Fig. 19. \# triples: partitions vs. summary.

the summarization performance for the SCHEMA method. The comparisons are shown in Figure 17. Regardless of the quality of the summary, the SCHEMA method in general performs an order of magnitude faster than our SUMm method across all the data sets we experimented. However, as it will become more clearly shortly, while the summary built by SCHEMA might be useful in some settings, it does not yield correct results in all our experimental data sets.

Size of the summary. As the SCHEMA method generates one (type) node in the summary for all the nodes in the data that have the same type, the size of summary (in terms of number of nodes) is equal to the number of distinct types from the data, as shown in Figure 14 For our summarization technique, we plot the number of partitions and the number of summaries in Figures 18(a) and 18(b) In Figure 18(a) for LUBM, the summarization technique results in at least two orders less 
distinct structures comparing to the number of partitions. Even in the extreme case where the dataset is partitioned into about a million subgraphs, the number of distinct summaries is still less than 100. In fact, it remains almost a constant after we increase the size of the data set to 1 million triples. This is because LUBM data is highly structured [10].

Not all RDF data sets are as structured as LUBM. Some RDF datasets like BSBM and DBpedia are known to have a high variance in their structuredness [10]. In Figure 18(b)] we plot the number of distinct summaries for other datasets after applying the SUMM method. For Wordnet and Barton, SUMM distills a set of summaries that has at least three orders less distinct structures than the respective set of partitions. Even in the case of DBpedia and BSBM, our technique still achieves at least one order less distinct structures than the number of the partitions, as shown in Figure 18(b).

In Figures 19(a) and 19(b), we compare the number of triples stored in the partitions and in the summary. Clearly, the results show that the distinct structures in the data partitions can be compressed with orders-of-magnitude less triples in the summary, e.g., at least one order less for DBpedia Infobox, and at least three orders less for LUBM, Wordnet, Barton and BSBM. Since the summaries are all small, this suggests that we can keep the summaries in main memory to process keyword query. Therefore, the first level of backward search for SUMM method can be mostly computed in memory.

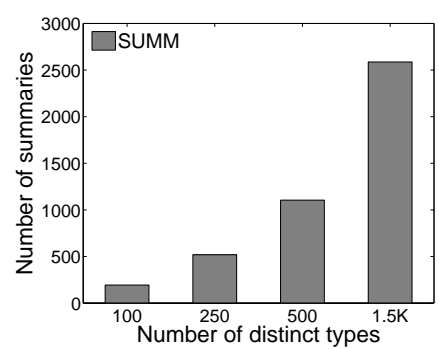

(a) \# summaries vs. \# types.

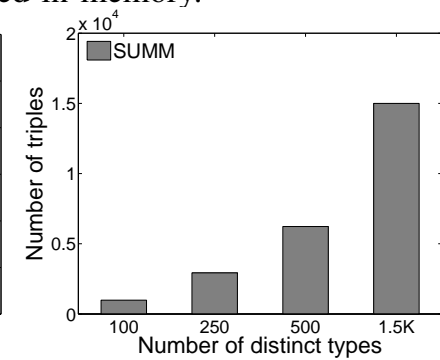

(b) \# triples vs. \# types
Fig. 20. Vary the number of types in BSBM.

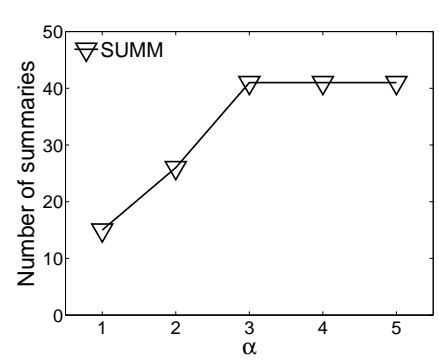

(a) LUBM

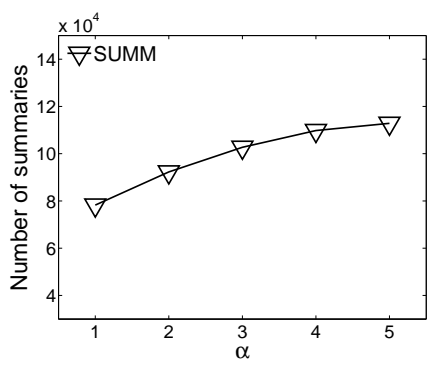

(b) DBPedia Infobox.
Fig. 21. Impact of $\alpha$ to the size of summary.

Impact from the distinct number of types. Adding new types of entities in an RDF data set implicitly adds more variances to the data and hence makes the summarization process more challenging. To see the impact on SUMM, we leverage the BSBM data generator. In particular, we generate four BSBM data sets with the number of distinct types ranged from 100 to 1,500 . The results from SUMM on these data sets are shown in Figures 20(a) and (b). As we are injecting more randomness into the data by adding more types, the size of the summary increases moderately. Consistent to our observations

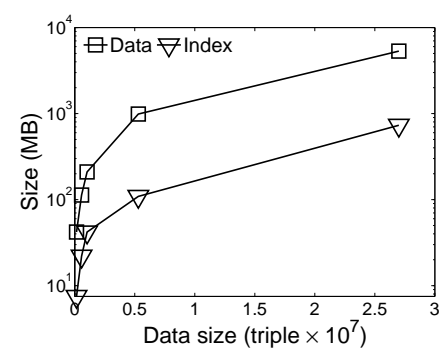

(a) LUBM.

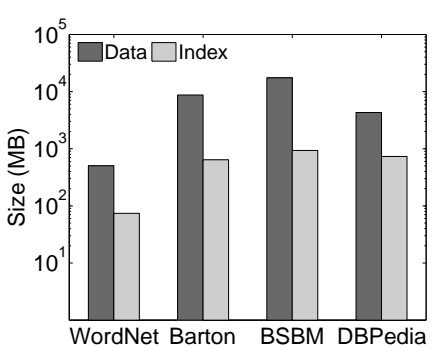

(b) Other datasets.
Fig. 22. Size of the auxiliary indexes.

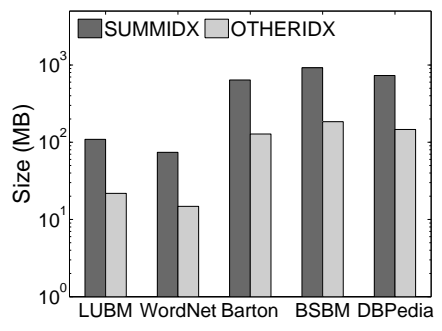

Fig. 23. Breakdown.

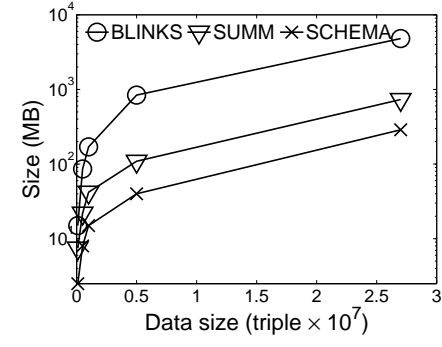

Fig. 24. Indexes on LUBM. for DBpedia in Figures 18(b) and 19(b), SUMM efficiently summarizes the BSBM data sets with orders less triples, even when the data set has more than a thousand distinct types.

Impact of $\alpha$. Since trends are similar, we only report the impact of $\alpha$ on two representative data sets, i.e., LUBM and DBpedia. In Figures 21(a) and 21(b) we report the impact of $\alpha$ (a parameter on the max number of hops in each partition, see Section 5.2 on the size of summary. Intuitively, the smaller $\alpha$ is, the more similar the $\alpha$-neighborhoods are, leading to a smaller size of summary after performing summarization. This is indeed the case when we vary $\alpha$ for all the data sets. The smallest summary is achieved when $\alpha=1$ in both Figures 21(a) and 21(b) Notice that there is a trade-off between the size of the summary and the size of the auxiliary indexes. A smaller partition implies that more nodes become portals, which increases the size of the auxiliary indexes. On the other hand, increasing $\alpha$ leads to larger partitions in general, which adds more variances in the structure of the partitions and inevitably leads to a bigger summary. However in practice, since the partitions are constructed by directed traversals on the data, we observed that most of the directed traversals terminate after a few hops. For instance, in LUBM, most partitions stop growing when $\alpha>3$. A similar trend is visible in Figures 21(b) When we increase $\alpha$, the number of distinct structures increases moderately.

Overheads of auxiliary indexes. In Figures 22(a) and 22(b) we study the size of the auxiliary indexes. Figure 22(a) shows that for LUBM, the size of the auxiliary indexes is one order less than the respective data when we vary its size up to 28 million triples. Similar trends can be observed in Figure 22(b) for the other datasets. This is because that for all indexes, we do not explicitly store the edges of the RDF data that usually dominate the cost in storing large graphs. In Figure 23, we report the breakdown of the inverted indexes for all the datasets. The most costly part is to store the mappings in the summary index (i.e., SUMMIDX in the figure) and the other indexes are all comparably small in size. Thus, to efficiently process query, 


\begin{tabular}{|c|c|c|}
\hline Query & \# nodes & Dataset \\
\hline $\mathrm{Q}_{1}\left[\mathrm{Pub}_{19}, \mathrm{Lec}_{6}\right]$ & $(20,13)$ & $\mathrm{L}$ \\
\hline $\mathrm{Q}_{2} \quad\left[\right.$ Research $_{5}$, FullProf 9, Pub $\left._{17}\right]$ & $(9,4,83)$ & $\mathrm{L}$ \\
\hline $\mathrm{Q}_{3} \quad\left[\right.$ FullProf $\left._{9}, \mathrm{Grad}_{0}, \mathrm{Pub}_{18}, \mathrm{Lec}_{6}\right]$ & $(4,15,40,5)$ & $\mathrm{L}$ \\
\hline $\mathrm{Q}_{4} \quad\left[\mathrm{Dep}_{0}, \mathrm{Grad}_{1}, \mathrm{Pub}_{18}\right.$, AssocProf $\left._{0}\right]$ & $(1,15,40,15)$ & $\mathrm{L}$ \\
\hline$Q_{5}$ [Afghan, Afghanistan, al-Qaeda, al-Qa'ida] & $(6,3,3,2)$ & $\mathrm{W}$ \\
\hline$Q_{6}\left[3^{\text {rd }}\right.$ base, $1^{\text {st }}$ base, baseball team, solo dance $]$ & $(14,13,17,4)$ & $\mathrm{W}$ \\
\hline$Q_{7} \quad$ [Knuth, Addison-Wesley, Number theory] & $(1,1,35)$ & $\mathrm{B}$ \\
\hline $\mathrm{Q}_{8}$ [Data Mining, SIGMOD, Database Mgmt.] & $(166,1,4)$ & $\mathrm{B}$ \\
\hline $\mathrm{Q}_{9} \quad$ [Bloomberg, New York City, Manhattan] & $(1,7,108)$ & $\mathrm{D}$ \\
\hline$Q_{10}[$ Bush, Hussein, Iraq] & $(1,1,48)$ & $\mathrm{D}$ \\
\hline $\mathrm{Q}_{11}$ [deflation, railroaders] & $(32,70)$ & $\mathrm{S}$ \\
\hline $\mathrm{Q}_{12}$ [ignitor, microprocessor, lawmaker] & $(3,110,43)$ & $\mathrm{S}$ \\
\hline
\end{tabular}

Fig. 25. Sample query workload

we can keep all but the summary index in main memory.

We also compare in Figure 24 the indexing overhead of different methods as we vary the data size for LUBM. Notice that BLINKS is the most costly method in terms of storage (i.e., it demands at least one order of magnitude more space), as its distance matrix leads to a quadratic blowup in indexing size. BLINKS is no doubt the faster method for small data, but it clearly does not scale with large RDF datasets. Therefore, we do not report BLINKS in the evaluation of query performance.

\subsection{Query performance}

In this section, we study the performance of top- $k$ keyword search using SUMM and BACKWARD. In particular, we compare the proposed methods with SCHEMA, which is the only existing method that can apply to large RDF datasets. To this end, we design a query workload that has various characteristics. Figure 25 lists 12 typical queries, together with the number of keyword occurrences in the datasets. For LUBM, all the keywords are selected from the first university in the data set, except for keywords with respect to publications 17 and 18. For the two indicated keywords, we select one copy of each publication from the first university and pick the rest of them randomly from other universities. This is to simulate the cases in real data sets where not all the keywords in a query are close to each other. Notice that in LUBM, keywords such as publications 17 or professor 9 are associated with multiple entities. For the other data sets, we pick two representative queries for each of them. The queries are shown in Figure 25. In particular, the second column \# nodes shows the number of occurrences for the keywords being queried in the respective data set. For long running queries, we terminate the executions after 1000 seconds. The response times are in log scale.

We first use SCHEMA to answer the queries in Figure 25. SCHEMA generates a set of $k$ SPARQL queries for each keyword query. Evaluating these queries are supposed to return $k$ answer roots ranked by the scores. However, even if we have fixed the incorrect termination condition in SCHEMA (as discussed in Section 4), our observation is that SCHEMA still returns incorrect results for all of the queries, as we have indicated in Figure 2. This can be explained by the way it summarizes the data, as all nodes of the same type are indistinguishably mapped to the same type node in the summary. For instance, every FullProfessor has a publication

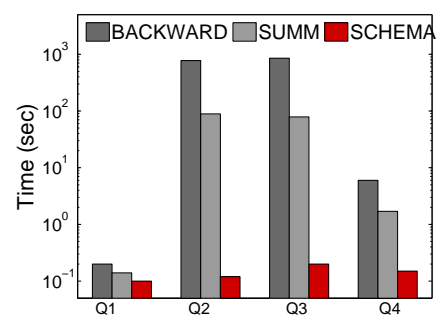

(a) LUBM

Fig. 26. Query performance.

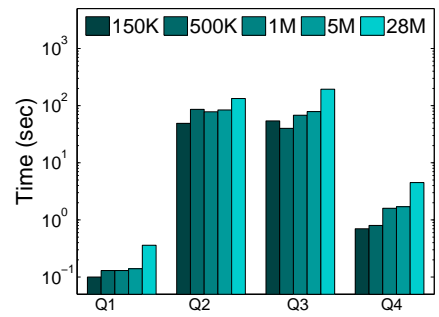

(a) SUMM

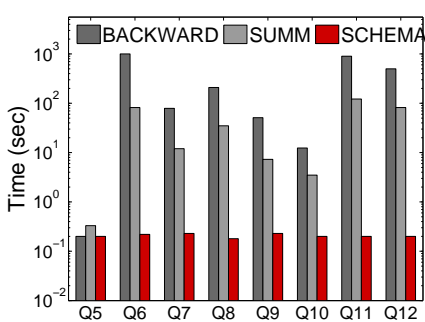

(b) Other datasets.

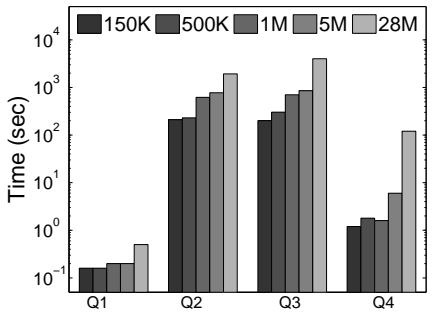

(b) BACKWARD
Fig. 27. Query performance vs. data size.

in LUBM, whereas this does not mean that FullProfessor9 has a Publication 17 for $Q_{2}$. Nevertheless, we report the response time of the SCHEMA method together with other methods for comparison. In what follows, we focus on discussing the query performance for the BACKWARD and SUMM methods, both of which have provable guarantees on the correctness of the query results. Their performance on the sampled query workload are plotted on Figures 26(a) and 26(b) respectively.

The efficiency of a keyword search is determined by a collection of factors [14], with no single factor being the most deterministic one. In particular, we observe that for selective keywords (i.e., keywords that have fewer occurrences in the data) and especially those that are close to each others, e.g., $Q_{1}, Q_{5}$, both BACKWARD and SUMM answer the queries efficiently. In some cases, e.g., $\mathrm{Q}_{5}$, BACKWARD outperforms Summ since Summ uses a lower bound to decide when to terminate. This inevitably requires SUMM to access more data than what is necessary to correctly answer the query.

However, being selective alone does not necessarily lead to better query performance, especially if the keyword being queried corresponds to a hub node that has a large degree, e.g., the Department0 in $\mathrm{Q}_{4}$. On the other hand, as the keywords being queried become non-selective, e.g., $\mathrm{Q}_{3}$ or $\mathrm{Q}_{11}$, or the keywords are far away from one another, e.g., the solo dance and the baseball team in $Q_{6}$, the SUMM approach generally performs much better than the BACKWARD method. This is because only when the connected partitions are found to contain all the keywords, the SUMM needs to access the whole subgraph on disk. This leads to savings in dealing with keywords that are resultless, e.g., most of the occurrences for publications 17 and 18 in $Q_{2}-Q_{4}$ fall into this category. In addition, at the partition level, the backward expansion in the SUMM approach can be done almost completely in memory as the major indexes for expansion are lightweight (as shown in Section 8.1 and therefore can be cached in memory for query evaluation. In such cases, i.e., $Q_{2}-Q_{4}$ and $Q_{6}-Q_{12}$, we observe that the SUMM approach performs much better.

In Figures 27 (a) and (b), we investigate the query perfor- 
mance while varying the size of the LUBM data set. As shown in the figure, for both SUMM and BACKWARD, the cost of query evaluation generally becomes more expensive as the size of the data increases. In contrast to BACKWARD, the difference in time for the SUMM method is relatively moderate as the size of the data changes. This is because for SUMM, keywords that are far apart can be pruned in the first level of the backward search. Unlike SUMM, BACKWARD has to pay equal effort in dealing with each copy of every keyword being queried, leading to a much slower convergence.

\section{CONCLUSION}

We studied the problem of scalable keyword search on big RDF data and proposed a new summary-based solution: (i) we construct a concise summary at the type level from RDF data; (ii) during query evaluation, we leverage the summary to prune away a significant portion of RDF data from the search space, and formulate SPARQL queries for efficiently accessing data. Furthermore, the proposed summary can be incrementally updated as the data get updated. Experiments on both RDF benchmark and real RDF datasets showed that our solution is efficient, scalable, and portable across RDF engines. An interesting future direction is to leverage the summary for optimizing generic SPARQL queries on large RDF datasets.

\section{ACKNOWLEDGEMENT}

Feifei Li was supported in part by NSF grant IIS-1251019.

\section{REFERENCES}

[1] S. Abiteboul, R. Hull, and V. Vianu. Foundations Of Databases. Addison-Wesley, 1995.

[2] A. Aggarwal and J. S. Vitter. The input/output complexity of sorting and related problems. CACM, 31(9):1116-1127, 1988.

[3] S. Agrawal, S. Chaudhuri, and G. Das. DBXplorer: enabling keyword search over relational databases. In SIGMOD, 2002.

[4] G. Bhalotia, A. Hulgeri, C. Nakhe, S. Chakrabarti, and S. Sudarshan. Keyword searching and browsing in databases using banks. In ICDE, 2002.

[5] C. Bizer and A. Schultz. The berlin SPARQL benchmark. International Journal On Semantic Web and Information Systems, 2009.

[6] J. Broekstra and et al. Sesame: A generic architecture for storing and querying RDF and RDF schema. In ISWC, 2002.

[7] Y. Chen, W. Wang, and Z. Liu. Keyword-based search and exploration on databases. In ICDE, 2011.

[8] Y. Chen, W. Wang, Z. Liu, and X. Lin. Keyword search on structured and semi-structured data. In SIGMOD, 2009.

[9] B. B. Dalvi, M. Kshirsagar, and S. Sudarshan. Keyword search on external memory data graphs. In $V L D B, 2008$.

[10] S. Duan, A. Kementsietsidis, K. Srinivas, and O. Udrea. Apples and oranges: a comparison of RDF benchmarks and real RDF datasets. In SIGMOD, 2011.

[11] $\mathrm{H}$. Fu and K. Anyanwu. Effectively interpreting keyword queries on rdf databases with a rear view. In ISWC, 2011.

[12] K. Golenberg, B. Kimelfeld, and Y. Sagiv. Keyword proximity search in complex data graphs. In SIGMOD, 2008.

[13] Y. Guo, Z. Pan, and J. Heflin. LUBM: A benchmark for OWL knowledge base systems. Journal of Web Semantics, 2005.

[14] H. He, H. Wang, J. Yang, and P. S. Yu. Blinks: ranked keyword searches on graphs. In SIGMOD, 2007.

[15] V. Hristidis, L. Gravano, and Y. Papakonstantinou. Efficient IR-style keyword search over relational databases. In $V L D B, 2003$.

[16] P. Jeavons. On the algebraic structure of combinatorial problems. Theoretical Computer Science, 1998.

[17] M. Kargar and A. An. Keyword search in graphs: Finding r-cliques. In $V L D B, 2011$.
[18] G. Li, B. C. Ooi, J. Feng, J. Wang, and L. Zhou. Ease: Efficient and adaptive keyword search on unstructured, semi-structured and structured data. In SIGMOD, 2008.

[19] Y. Luo, W. Wang, and X. Lin. SPARK: A keyword search engine on relational databases. In ICDE, 2008.

[20] A. Polleres. From SPARQL to rules (and back). In WWW, 2007.

[21] R. Shamir and D. Tsur. Faster subtree isomorphism. Journal of Algorithms, 1999.

[22] C. Sun, C. Y. Chan, and A. K. Goenka. Multiway slca-based keyword search in xml data. In $W W W$, pages 1043-1052, 2007.

[23] T. Tran, H. Wang, S. Rudolph, and P. Cimiano. Top-k exploration of query candidates for efficient keyword search on graph-shaped (RDF) data. In ICDE, 2009.

[24] C. Weiss, P. Karras, and A. Bernstein. Hexastore: sextuple indexing for semantic web data management. PVLDB, 2008.

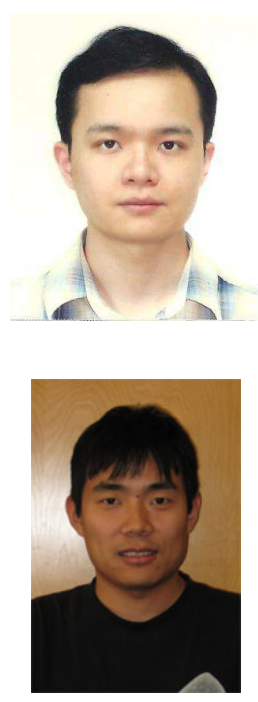

Wangchao Le holds a PhD degree from the University of Utah, specialized in data management and analysis. Wangchao's research interests lie in database systems, in particular, temporal and multi-version data, query processing and optimization for large graph data, and semantic web. His dissertation is about supporting scalable data analytics on large linked data. Wangchao is currently working at Microsoft for its big data and search infrastructure.

Feifei $\mathrm{Li}$ received the BS degree in computer engineering from the Nanyang Technological University in 2002 and the $\mathrm{PhD}$ degree in computer science from the Boston University in 2007. He is currently an associate professor in the School of Computing, University of Utah. His research interests include database and data management systems and big data analytics.

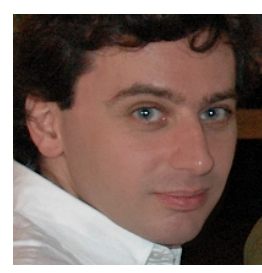

Anastasios Kementsietsidis is a Research Staff Member at IBM T. J. Watson Research Center. He has a $\mathrm{PhD}$ from the University of Toronto. Anastasios is currently interested in various aspects of RDF data management (including, querying, storing and benchmarking RDF data). He is also interested in data integration, cleaning, provenance and annotation, as well as (distributed) query evaluation and optimization on relational or semi-structured data.

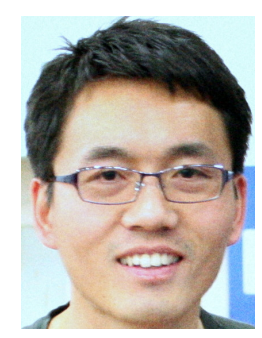

Songyun Duan is a researcher at IBM T. J. Watson Research Center. His research interests lie in data management, machine learning, bigdata analysis, and semantic web. Songyun got his PhD degree from Duke University, with a dissertation on simplifying system management through automated forecasting, diagnosis, and configuration tuning. 


\section{Online Appendix To TKDE Submission "Scalable Keyword Search on Large RDF Data" by Wangchao Le, Feifei Li, Anastasios Kementsietsidis, Songyun Duan}

\section{SPARQL QUERY}

SPARQL, a W3C recommendation, is a pattern-matching query language. For instance, to extract the vertices that are connected by predicates launchPad and booster in Figure 1, one could issue a SPARQL query of two conjunctive triple patterns as in Figure 28. The actual bindings for the variables (whose names begin with "?") will be retrieved by evaluating the query on the dataset. Notice that there is an optional triple pattern in the query, where its matching is not enforced in the evaluation of the query. Evaluating the query on the data in Figure 11 will retrieve two spacemissions, ? $x=\mathrm{URI}_{3}$ and $? x=\mathrm{URI}_{5}$. Note that the state-of-the-art RDF stores do not

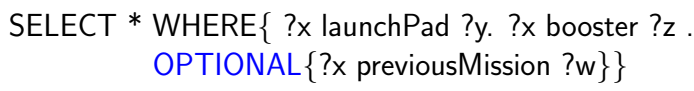

Fig. 28. A SPARQL query.

support keyword queries, especially for keyword queries that try to find subgraphs connecting all the query keywords.

\section{PROOFS}

Lemma $1 g=\left\{r=u, v_{\ell_{1}}, \ldots, v_{\ell_{m}}\right\}$ is a candidate answer with $s(g)=\sum_{i=1}^{m} d\left(u, v_{\ell_{i}}\right)$.

Proof: By our construction for entries in $M, w\left(v_{\ell_{i}}\right)=w_{i}$ and $d\left(u, v_{\ell_{i}}\right)$ is the length for shortest path from $v_{\ell_{i}}$ to $u$, which completes the proof.

Lemma 2 Let $g_{1}$ be the best possible candidate answer, with $v \notin M$ being the root node of $g_{1}$. Then $s\left(g_{1}\right)>\sum_{i=1}^{m} d\left(p_{i}\right)$.

Proof: Since $v$ is not in $M$, indicating that $v$ has not yet been included in any expansion path from any entries in these $m$ queues, we need to expand at least one neighboring node to the end node in a path from at least one of these top $m$ entries to possibly reach $v$. Furthermore, all $m$ expansion queues sort their entries in ascending order of the distance of the corresponding paths, hence, any candidate answer using $v$ as the root node must have at least a distance of $d\left(p_{i}\right)+1$ to reach a vertex $v^{\prime}$ with $w\left(v^{\prime}\right)=w_{i}$. That shows $s\left(g_{1}\right)>$ $\sum_{i=1}^{m} d\left(p_{i}\right)$, which completes the proof.

Lemma 3 Suppose the best possible candidate answer using such an $v \in M$ as the root node is $g_{2}$, then

$$
s\left(g_{2}\right)>\sum_{i=1}^{m} f\left(v_{b_{i}}\right) d_{i}+\left(1-f\left(v_{b_{i}}\right)\right) d\left(\mathrm{p}_{\mathrm{i}}\right),
$$

where $f\left(v_{b_{i}}\right)=1$ if $M[v]\left[b_{i}\right] \neq n i l$, and $f\left(v_{b_{i}}\right)=0$ otherwise.

Proof: When $v_{b_{i}}$ is not nil, that means this vertex $v$ has been reached by an expansion from a vertex $v_{b_{i}}$ where $w\left(v_{b_{i}}\right)=w_{i}$ (i.e.,,$\left.v_{b_{i}} \in W_{i}\right)$, and $d_{i}=d\left(v_{b_{i}}, v\right)$. More importantly, $d_{i}$ is the shortest possible distance from any vertex in $W_{i}$ to reach $v$, since we expand paths in $a_{i}$ in the ascending order of their distances.
When $v_{b_{i}}$ is nil, that means no expansions initiated from any vertices from $W_{i}$ has yet reached $v$. Following the same argument from the proof for Lemma 2, the shortest distance to reach any vertex in $W_{i}$ from $v$ is at least $d\left(p_{i}\right)+1$.

Finally, by combining these two arguments, we can establish Equation (2).

Theorem 1 The BACKWARD method finds the top- $k$ answers $\mathcal{A}(q, k)$ for any top- $k$ keyword query $q$ on a RDF graph.

Proof: This is a straightforward result by Lemma 1 and the termination conditions stated in Lemmas 2, 3.

Lemma 4 Partitions in $\mathcal{P}$ are edge disjoint and the union of all partitions in $\mathcal{P}$ cover the entire graph $G$.

Proof: The edge disjoint property trivially holds by our construction of $h(v, \alpha)$. By visiting the vertices in each type, we have effectively included the $\alpha$-neighborhoods of all vertices in $G$ into $\mathcal{P}$, which leads to the conclusion that the union of the resulting partitions covers $G$.

Lemma 5 Given two graphs $g$ and $h$, if $f: g \rightarrow h$, then $\forall v_{1}$, $v_{2} \in g$ and their homomorphic mappings $f\left(v_{1}\right), f\left(v_{2}\right) \in h$, $d\left(v_{1}, v_{2}\right) \geq d\left(f\left(v_{1}\right), f\left(v_{2}\right)\right)$.

Proof: By definition, $\forall(u, v) \in g,(f(u), f(v)) \in h$. Since every edge in $g$ is mapped to an edge in $h$ by homomorphism, the shortest path $p$ that leads to $d\left(v_{1}, v_{2}\right)$ in $g$ can be mapped to a path in $h$ that starts at $f\left(v_{1}\right)$ and ends at $f\left(v_{2}\right)$ by applying $f$ on each of the edges on $p$. Thus, $d\left(f\left(v_{1}\right), f\left(v_{2}\right)\right)$ is at most $d\left(v_{1}, v_{2}\right)$.

Lemma 6 For a partition $h$ and its covering tree $h_{t}$, there is a homomorphism from $h$ to Join $\left(h_{t}, \Sigma\right)$.

Proof: We construct such a homomorphic function $f$ : $h \rightarrow \operatorname{Join}\left(h_{t}, \Sigma\right)$. Notice that by the objective of building summaries, we only consider the types of nodes. For a node $v \in h$, if it is not registered by any mapping $\sigma \in \Sigma$, i.e., it does not appear in two different paths, then let $f(v)=v$, since $\operatorname{Join}\left(h_{t}, \Sigma\right)$ has no effect on $v$; else if a node $v$ is registered by some $\sigma_{i} \in \Sigma$, then by the property of Join, all vertices in $h_{t}$ that have the type $\sigma_{i}(v)$ will be combined into one node. Let this node be $u$, then $f(v)=u$. Now consider the edges in $h$, by construction, $h_{t}$ records all the paths from the partition root in $h$ to every other node in $h$ and Join does not add or delete edges or change their labels. Thus, if there is an edge $\left(v_{1}, v_{2}\right) \in h$, then there is an edge in $h_{t}$ with the same label and the same types of the starting and ending nodes in $h_{t}$. Since Join will not alter the type of a node as well, it follows $\left(f\left(v_{1}\right), f\left(v_{2}\right)\right)$ must be an edge in Join $\left(h_{t}, \Sigma\right)$ with the same label and the same starting and ending types. 
Lemma 7 For a partition $h$, its covering tree $h_{t}$ and its summary $s$ that has $f_{2}: h_{t} \rightarrow s$, there is a homomorphism from Join $\left(h_{t}, \Sigma\right)$ to Join $\left(s, f_{2}(\Sigma)\right)$.

Proof: We will construct such a homomorphism by the function $f: \operatorname{Join}\left(h_{t}, \Sigma\right) \rightarrow \operatorname{Join}\left(s, f_{2}(\Sigma)\right)$.

For a node $v \in h_{t}$, consider the correspondent $f_{2}(v)$ in $s$. If $v$ is not registered by any $\sigma \in \Sigma$, then $\operatorname{Join}\left(h_{t}, \Sigma\right)$ has no effect on $v$ and $\operatorname{Join}\left(s, f_{2}(\Sigma)\right)$ has no effect on $f_{2}(v)$, hence $v \in \operatorname{Join}\left(h_{t}, \Sigma\right)$ and $f_{2}(v) \in \operatorname{Join}\left(s, f_{2}(\Sigma)\right)$. Define $f=f_{2}$ for such $v$ 's. If $v$ is in some $\sigma_{i} \in \Sigma$, all nodes in $h_{t}$ that have the same type as $v$ will be combined into one node. Let this node be $u$. On the other hand, by Join $\left(s, f_{2}(\Sigma)\right)$, all nodes that have the same type as $f_{2}(v)$ will be combined into one node. Let this node be $u^{\prime}$. Define $f(u)=u^{\prime}$. Now consider the edges. Notice that Join has no effect on edges and for every $\left(v_{1}, v_{2}\right) \in h_{t},\left(f_{2}\left(v_{1}\right), f_{2}\left(v_{2}\right)\right) \in s$. This follows that for every $\left(f\left(v_{1}\right), f\left(v_{2}\right)\right) \in \operatorname{Join}\left(h_{t}, \Sigma\right)$, there is an edge $\left(f\left(f_{2}\left(v_{1}\right)\right), f\left(f_{2}\left(v_{2}\right)\right)\right) \in \operatorname{Join}\left(s, f_{2}(\Sigma)\right)$.

Lemma 8 Let $\left(v,\left(u, \mathcal{S}, d_{l}, d_{u}\right)\right)$ be an entry in the priority queue, then for any $v^{\prime}$ in the partition rooted at $v$ and for any path starting from $u$ and using the portals in $\mathcal{S}$, its length $d\left(u, v^{\prime}\right) \geq d_{l}$.

Proof: Let $\mathcal{S}=\left\{\left(\ell_{1}, v_{1}\right),\left(\ell_{2}, v_{2}\right), \ldots,\left(\ell_{k}, v_{k}\right)\right\}$. It has $d\left(u, v^{\prime}\right) \geq d\left(u, \ell_{k}\right)+d\left(\ell_{k}, v^{\prime}\right) \geq d\left(u, \ell_{k}\right)$, where $\ell_{k}$ is the portal in $\mathcal{S}$ that the path uses to enter the partition rooted at $v$ and $d\left(u, \ell_{k}\right)$ is the length for the sub-path that reaches the portal $\ell_{k}$ from $u$. Let $d\left(\ell_{i}, \ell_{i+1}\right)$ be the fragment of the path that is in the partition rooted at $v_{i+1}(i=0, \ldots, k-1)$ and $\ell_{0}=u$, we have $d\left(u, \ell_{k}\right)=d\left(u, \ell_{1}\right)+d\left(\ell_{1}, \ell_{2}\right)+\ldots+$ $d\left(\ell_{k-1}, \ell_{k}\right) \geq d_{l}\left(u, \ell_{1}\right)+d_{l}\left(\ell_{1}, \ell_{2}\right)+\ldots+d_{l}\left(\ell_{k-1}, \ell_{k}\right)=d_{l}$.

Lemma 9 Let $\left(v,\left(u, \mathcal{S}, d_{l}, d_{u}\right)\right)$ be the top entry in the priority queue $a_{i}$, then for any explored path $p$ from $w_{i}$ in the queue $a_{i}$, the length of $p$, written as $d(p)$, has $d(p) \geq d_{l}$.

Proof: Let $\left(v^{\prime},\left(u^{\prime}, \mathcal{S}^{\prime}, d_{l}^{\prime}, d_{u}^{\prime}\right)\right)$ be any entry in $a_{i}$ that represents a path starting at $u^{\prime}$ and reaches partition rooted at $v^{\prime}$. Denote this path as $p^{\prime}$ and its length as $d\left(p^{\prime}\right)$. From Lemma $8, d\left(p^{\prime}\right) \geq d_{l}^{\prime}$. By the property of priority queue (minheap), $d_{l}^{\prime} \geq d_{l}$ for any entry in $a_{i}$.

Lemma 10 Let $g_{1}$ be the possible unexplored candidate answer rooted at a vertex in partition $h$, with $h \in \mathcal{P}_{t}$,

$$
s\left(g_{1}\right)>\sum_{i=1}^{m} d_{l}^{i} .
$$

Proof: Since $h \notin M$, in order to reach $h$, we need at least one expansion from some partition to its neighboring partition from at least one of the top $m$ entries in the priority queues. By Lemma 9, the length for the shortest possible path from a keyword $w_{i}$ is lower bounded by $d_{l}^{i}$. Therefore, to reach partition $h$, it requires a distance of at least $d_{l}^{i}+1$. This shows $s\left(g_{1}\right)>\sum_{i=1}^{m} d_{l}^{i}$.
Lemma 11 Let the best possible unexplored candidate answer as $g_{2}$, which is rooted at a vertex in the partition $h$, where $h \in \mathcal{P}-\mathcal{P}_{t}$, then

$$
s\left(g_{2}\right)>\sum_{i=1}^{m} f\left(t_{i}\right) \hat{d}_{l}^{i}+\left(1-f\left(t_{i}\right)\right) d_{l}^{i},
$$

where $f\left(t_{i}\right)=1$ if $t_{i} \neq$ nil otherwise $f\left(t_{i}\right)=0$.

Proof: The candidate subgraph formed by $\left(t_{1}, \ldots, t_{m}\right)$ from the entry $h$ in $M$ has the smallest (aggregated) lower bound from combining the lower bounds in all $m$ lists, due to the fact that each list is sorted in ascending order by the lower bound distance $d_{l}$.

When some $t_{i}=$ nil, no expansion from any vertex associated with the keyword $w_{i}$ has reached the partition $h$ yet. Following the same argument from the proof for Lemma 10. the shortest distance to reach a vertex in $W_{i}$ is at least $d_{l}^{i}+1$. For the others where $t_{i} \neq n i l$, the best possible unexplored path from any keyword $w_{i}$ to reach some node in $h$ will have a distance that is no less than $\hat{d}_{l}^{i}$ due to the property of the priority queue and the fact that it is a lower bound.

Theorem 2 The SUMM method correctly finds the top- $k$ answers $\mathcal{A}(q, k)$ for any top- $k$ keyword search query $q$ on an RDF graph.

Proof: By combining Lemmas 10 and 11, it suffices to derive the termination condition. Together with Theorem 1 , it guarantees the correctness of the SUMM method.

\section{Complexity}

Our analysis will be carried out in the standard external memory model of computation [2]. In this model, a disk has been formatted into blocks of size $B$. An I/O operation either reads a block from the disk to memory, or conversely, writes a block in memory to the disk. Under the external memory model, we analyze the complexity of the SUMM method in the number of I/Os.

Unlike the problems defined in [4], [17] where their objectives amount to finding a minimum Steiner tree, the problem we have settled in this paper is tractable (see eq 1). The same definition has also been adopted in [14], [23]. For any keyword query $q=\left\{w_{1}, w_{2}, \ldots, w_{m}\right\}$, denote the total number matches for $w_{i} \mathrm{~s}$ in the data graph $G$ as $T$. For a valid query $q$, i.e., $w_{i} \in G(i=1 . . m)$, we have $T \geq m$ (since a keyword $w_{i}$ might appear more one time in $G$ ); otherwise $\exists w_{i} \notin G$, the query is invalid and our method returns in $O(m)$ I/Os after checking the inverted indexes. Hereafter, we focus our discussion on the first case, where the query $q$ is valid.

In the worst case, the whole data set $G$ is useful in finding the best score and the baseline BACKWARD method has to traverse every triple in $G$ for each of the $T$ copies of the matched keywords, i.e., incurring $T$ backward expansions and leading to a overall cost of $O(T \cdot|E| / B)$ I/Os. We dab this cost $O(\operatorname{WORST}(T, E))$. To save the cost spent on the expansions that are resultless, the SUMM method employs a two-level branchand-bound procedure to answer the keyword query. Assume that the summaries are small enough such that they can be 
kept in memory for query evaluation (see section 8 for the empirical evaluations on this assumption). On the first level of backward expansion, SUMM method identifies and connects partitions that are promising in answering $q$. This amounts to retrieving the portals and the triples in the relevant partitions. Denote the fraction of vertexes accessed in retrieving the portals as $0<\sigma \leq 1$, and the fraction of triples accessed for reconstructing the partitions as $0<\gamma \leq 1$. The second level of backward expansion then performs search on the identified partitions, calculates the minimum cost(s) and looks for the best answer $\operatorname{root}(\mathrm{s})$, all of which can be done in memory, e.g., the exact distance between portals across partitions can be computed incrementally. Therefore, the dominant cost for the SuMm method is from the first level of backward search, which has a cost of $O(\sigma \cdot|V| / B+\gamma \cdot T \cdot|E| / B)$ I/Os. The actual values for $\sigma$ and $\gamma$ are data and query dependent. We dab this cost $O(\mathrm{OPT}(\sigma, V, \gamma, E))$. Therefore, the SUMM method outperforms the BACKWARD method if $O(\operatorname{OPT}(\sigma, V, \gamma, E))<$ $O(\operatorname{WORST}(T, E))$.

\section{MORE ON CORRECTNESS}

Regarding the correctness of the solution framework, we have rigorously shown in Theorem 2 that the SUMM method correctly finds the Top- $k$ answers for a given keyword query. There is another angle to examine the correctness of the SUMM method.

Let us first consider the case where the summary is not used in Algorithm 4. It is worth pointing out that the SUMM method in this case can still produce the correct Top- $k$ answers without using the summary. To achieve this, we can simply twist two lines in Algorithm 4. Firstly, in line 13, we always set the lower bound to zero and the upper bound to infinity. By doing so, the first level of backward search will eventually connect all possible partitions by the using the portal index; secondly, in line 19, we can simply use brute-force search to find the subgraph $g$ in the RDF data. Regardless of its performance, it is not hard to see that this solution produces correct answers as the SUMM method degenerates to a brute-force backward search.

Then, consider the case where the summary and the summary index are employed for early termination and a better efficiency. By using Lemmas 5, 6, 7, and the triangle inequality, we have shown that line 13 in Algorithm 4 is correct. By Lemmas 8,9 and 10 , we have shown the termination condition in Algorithm 4 is correct. The concern for the correctness of the SUMM method will diminish if we can show that line 19 is correct. This amounts to showing that the SPARQL query compiled from the summary can indeed retrieve all the data in every possible partition. Recall that there is a homomorphism from the covering tree $h_{t}$ to the original partition $h$, and there is a second homomorphism from the core $c$ of $h_{t}$ to $h_{t}$ (by the definition of a core). Therefore, $c$ is homomorphic to the original partition $h$. By Theorem 3 , the SPARQL query that uses $c$ as its search pattern can retrieve the partition $h$ from the RDF data set. If the SPARQL query does use $c$ as its search pattern to get the original partition $h$ from the data, then the correctness of the SUMM method is justified. To compile such a SPARQL query, we first use the summary index to find a summary node $v_{r}$ in some summary $s$ that corresponds the partition root $r$ of $h$ ( $c$ has been embedded in $s$ ); then by construction, the whole subtree $t$ rooted at $v_{r}$ in $s$ is used as the query pattern to (indirectly) retrieve $h$. Notice that $t$ is a supergraph of $c$ and the root of $c$ colocates with the root of $t$. By construction, level by level, we express each triple pattern of $t$ below $v_{r}$ in a (nested) OPTIONAL clause (i.e., left-outer join). Consider the root $v_{r}$ and its $n$ children, the fragment of the search pattern contains $n$ OPTIONAL clauses, each for one of the children (see Figure 13). By the property of left-outer join, evaluating this fragment of query against the RDF data set yields results that correspond to $2^{n}$ different combinations of the children. By induction, using (nested) OPTIONAL clauses can express all possible search patterns rooted at $v_{r}$. Therefore, the results by using the core $c$ as the search pattern can be retrieved. This completes the proof.

Finally, notice that although the query pattern we constructed from the summary is always a tree, the actual subgraphs in the data that match the search pattern could have arbitrary structures - as long as there is a homomorphism from the search pattern to the respective subgraph in the data. Thanks to Theorem 3, the SPARQL engine can fetch all the homomorphic subgraphs from the data.

\section{Dealing With updates}

One important limitation of previous work on summarizing RDF data is their inability to handle updates in the data in an incremental way. Here, we show that our summaries can be incrementally updated. In more details:

Insertion. Insertions can be handled efficiently. A new subgraph (a set of triples) is simply treated as a data partition that has not been traversed. Indexing structures and the summary can be updated accordingly.

Deletion. Let $t$ be the triple deleted. Then all the partitions that visit the subject/object of $t$ will be updated. As a deletion only affects the nodes in the $\alpha$-neighborhood of $t$ 's subject and object, this can be done efficiently. To update $S$, there are two cases to consider: (i) if the core of an updated partition is not in $S$, i.e., it is homomorphic to a core in $S$, we simply rebuild its core and update the corespondent inverted indexes; (ii) if the core of an updated partition is in $S$, this will lead to a removal for the core in $S$. In addition, we retrieve all the partitions homomorphic to the deleted core and summarize them (together with the updated partition) as if they are new data. To access these partitions efficiently, we can leverage the technique in Section 7 and use the (to be) deleted core as the query pattern. 